\title{
PLANE NON-SINGULAR CURVES WITH AN ELEMENT OF "LARGE" ORDER IN ITS AUTOMORPHISM GROUP
}

\author{
ESLAM BADR AND FRANCESC BARS
}

\begin{abstract}
Let $M_{g}$ be the moduli space of smooth, genus $g$ curves over an algebraically closed field $K$ of zero characteristic. Denote by $M_{g}(G)$ the subset of $M_{g}$ of curves $\delta$ such that $G$ (as a finite non-trivial group) is isomorphic to a subgroup of $A u t(\delta)$ and let $\widetilde{M_{g}(G)}$ be the subset of curves $\delta$ such that $G \cong A u t(\delta)$ where Aut $(\delta)$ is the full automorphism group of $\delta$. Now, for an integer $d \geq 4$, let $M_{g}^{P l}$ be the subset of $M_{g}$ representing smooth, genus $g$ plane curves of degree $d$ (in this case, $g=(d-1)(d-2) / 2)$ and consider the sets $M_{g}^{P l}(G):=M_{g}^{P l} \cap M_{g}(G)$ and $\widehat{M_{g}^{P l}(G)}:=\widetilde{M_{g}(G)} \cap M_{g}^{P l}$.

In this note we first determine, for an arbitrary but a fixed degree $d$, an algorithm to list the possible values $m$ for which $M_{g}^{P l}(\mathbb{Z} / m)$ is non-empty where $\mathbb{Z} / m$ denotes the cyclic group of order $m$. In particular, we prove that $m$ should divide one of the integers: $d-1, d, d^{2}-3 d+3,(d-1)^{2}, d(d-2)$ or $d(d-1)$. Secondly, consider a curve $\delta \in M_{g}^{P l}$ with $g=(d-1)(d-2) / 2$ such that $A u t(\delta)$ has an element of "very large" order, in the sense that this element is of order $d^{2}-3 d+3,(d-1)^{2}, d(d-2)$ or $d(d-1)$. Then we investigate the groups $G$ for which $\delta \in \widehat{M_{g}^{P l}(G)}$ and also we determine the locus $\widehat{M_{g}^{P l}(G)}$ in these situations. Moreover, we work with the same question when $\operatorname{Aut}(\delta)$ has an element of "large" order $\ell d, \ell(d-1)$ or $\ell(d-2)$ with $\ell \geq 2$ an integer.
\end{abstract}

\section{INTRODUCTION}

It is well known that any $\delta \in M_{g}^{P l}(G)$ corresponds to a set $\left\{C_{\delta}\right\}$ of non-singular plane models in $\mathbb{P}^{2}(K)$ such that any two of them are $K$-isomorphic through a projective transformation $P \in P G L_{3}(K)$ (where $P G L_{3}(K)$ is the classical projective linear group of $3 \times 3$ invertible matrices over $K$ ), and their automorphism groups are conjugate. If $C$ is a non-singular plane model of $\delta$ which is defined by the homogenous equation $F(X ; Y ; Z)=0$ then $\operatorname{Aut}(C)$ is a finite subgroup of $P G L_{3}(K)$ and also we have $\rho(G) \preceq A u t(C)$ for some injective representation $\rho: G \hookrightarrow P G L_{3}(K)$. Moreover, $\rho(G)=A u t(C)$ whenever $\delta \in \widetilde{M_{g}^{P l}(G)}$.

We denote by $\rho\left(M_{g}^{P l}(G)\right)$ the set of all elements $\delta \in M_{g}^{P l}(G)$ such that $G$ acts on a plane model associated to $\delta$ as $P^{-1} \rho(G) P$ for some $P$ inside $P G L_{3}(K)$. This gives us the following disjoint union decomposition:

$$
M_{g}^{P l}(G)=\cup_{[\rho] \in A} \rho\left(M_{g}^{P l}(G)\right)
$$

where $A:=\left\{\rho \mid \rho: G \hookrightarrow P G L_{3}(K)\right\} / \sim$ such that $\rho_{a} \sim \rho_{b}$ if and only if $\rho_{a}(G)=P^{-1} \rho_{b}(G) P$ for some $P \in P G L_{3}(K)$. A similar decomposition follows for $\widehat{M_{g}^{P l}(G)}$.

For a fixed degree $d$, it is a difficult task to list the $[\rho]^{\prime} s$ and the groups $G$ such that $\rho\left(M_{g}^{P l}(G)\right)$ is non-empty, see Henn work [9] and Komiya-Kuribayashi work [12] for degree 4 and 2] for degree 5. For a cyclic group $G \cong \mathbb{Z} / m$ of order $m$, Dolgachev in $\left[5\right.$ ] determined the $[\rho]^{\prime} s$ and $m$ such that $\rho\left(M_{3}^{P l}(\mathbb{Z} / m \mathbb{Z})\right)$ is non-trivial and moreover he associated to such locus (once $\rho$ and $m$ are fixed), a normal form, i.e. a certain projective equation which depends on some parameters together with some algebraic restrictions to these parameters such that any element of the locus $\rho\left(M_{g}^{P^{l}}(\mathbb{Z} / m \mathbb{Z})\right)$ corresponds to certain specialization of the parameters. In $\S 2$, following Dolgachev technique, we obtain a general algorithm in order to determine $[\rho]^{\prime} s$ and $m$ such that $\rho\left(M_{g}^{P l}(\mathbb{Z} / m \mathbb{Z})\right)$ might be non-trivial and also to such locus (once $\rho$ and $m$ are fixed) we associate a normal form (see Remark 7 for a link to an implementation of the algorithm in SAGE, and the appendix for listing the results that are given by the algorithm until degree 9). As a consequence of the algorithm (Theorem 6) we obtain that $m$

2010 Mathematics Subject Classification. 14H37, 14H50, 14H45.

Key words and phrases. non-singular curves; plane models; automorphism groups; moduli spaces.

E. Badr and F. Bars are supported by MTM2013-40680-P. 
always divides one of the following integers: $d^{2}-3 d+3,(d-1)^{2}, d(d-2)$ or $d(d-1)$, which we believe that is well-known to the specialists.

Secondly, there is a lot of interest on non-singular curves having a large automorphism group: For $K=\mathbb{C}$ a curve $\delta \in M_{g}$ has large automorphism group if it has a neighborhood (with respect to the complex topology) in $M_{g}$ such that any other curve inside the neighborhood has a smaller automorphism group. For such situations $\delta$ admits a model defined over $\mathbb{Q}, \delta / A u t(\delta)$ corresponds to the projective line and the Galois $\operatorname{cover} \delta \rightarrow \delta / A u t(\delta)$ is a Belyi morphism, in particular it is ramified exactly at 3 points (the last property of a Belyi morphism that is ramified at three points and is a Galois cover, characterizes curves with large automorphism group). For more details, we refer to Wolfart 14. Another notion in the literature for $\delta$ to be of large automorphism group is assuming that $|A u t(\delta)|>4(g-1)$. In particular, for $\delta \in M_{g}^{P l}$ it means that $|A u t(\delta)|>2\left(d^{2}-3 d+2\right)-4($ in this case $\delta \rightarrow \delta / A u t(\delta)$ is a map from $\delta$ to a projective line which is ramified at 3 or 4 points, see [6, p.258-260]).

The above definitions of large automorphism group are very restrictive to our proposes of plane curves $\delta \in M_{g}^{P l}$ and in this paper we say that an element $\sigma \in A u t(\delta)$ is "very large" if its order is exactly one of the integers $d^{2}-3 d+3,(d-1)^{2}, d(d-2)$ or $d(d-1)$. We say that $\sigma \in A u t(\delta)$ is "large" if its order is exactly one of the following integers: $\ell d, \ell(d-1)$ or $\ell(d-2)$ for some integer $\ell \geq 2$.

In what follows $\xi_{m}$ denotes a primitive $m$-th root of unity in $K$ and we obtain, in particular, the following results (in $\S 3.1$ to $\S 3.4)$ for $\delta \in M_{g}^{P l}(\mathbb{Z} / m)$ such that $m$ is "very large" in the above sense.

Theorem 1. Let $\delta \in M_{g}^{P l}$ be a non-singular plane curve of degree $d \geq 4$ and let $\sigma \in$ Aut $(\delta)$ where $\sigma$ is "very large". Then one of following cases occurs.

(1) if $\sigma$ has order $d(d-1)$ with $d \geq 5$ then Aut $(\delta)=<\sigma>$ and $\delta$ is $K$-isomorphic to $X^{d}+Y^{d}+X Z^{d-1}=0$. In particular for $d \geq 5, M_{g}^{P l}(\mathbb{Z} / d(d-1) \mathbb{Z})$ is an irreducible locus with one element, and

$$
\left.M_{g}^{P l}(\widetilde{\mathbb{Z} / d(d}-1)\right)=M_{g}^{P l}(\mathbb{Z} / d(d-1))=\rho\left(M_{g}^{P l}(\mathbb{Z} / d(d-1))\right)
$$

where $\rho(\mathbb{Z} / d(d-1) \mathbb{Z})=<\operatorname{diag}\left(1, \xi_{d(d-1)}^{d-1}, \xi_{d(d-1)}^{d}\right)>$. For the case $d=4$, one can read Remark 12 in $\S 3.1$ for further details.

(2) if $\sigma$ has order $(d-1)^{2}$ then Aut $(\delta)=<\sigma>$ and $\delta$ is $K$-isomorphic to $X^{d}+Y^{d-1} Z+X Z^{d-1}=0$. Also, $M_{g}^{P l}\left(\mathbb{Z} /(d-1)^{2} \mathbb{Z}\right)$ is an irreducible locus with one element, and

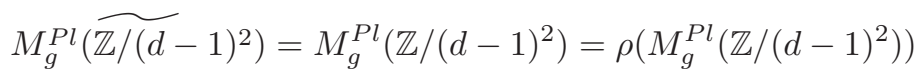

with $\rho\left(\mathbb{Z} /(d-1)^{2} \mathbb{Z}\right)=<\operatorname{diag}\left(1, \xi_{(d-1)^{2}}, \xi_{(d-1)^{2}}^{(d-1)(d-2)}\right)>$.

(3) if $\sigma$ has order $d(d-2)$ then $\delta$ is $K$-isomorphic to $X^{d}+Y^{d-1} Z+Y Z^{d-1}=0$ and for $d \neq 4,6$ we have

$$
H_{d}:=\operatorname{Aut}(\delta)=<\sigma, \tau \mid \tau^{2}=\sigma^{d(d-2)}=1 \text {, and } \tau \sigma \tau=\sigma^{-(d-1)}>\text {. }
$$

Again, $\left.M_{g}^{P l}(\mathbb{Z} / d(d-2) \mathbb{Z})\right)$ is an irreducible locus with one element, and

$$
\widetilde{M_{g}\left(H_{d}\right)}=M_{g}^{P l}(\mathbb{Z} / d(d-2))=\rho\left(M_{g}^{P l}(\mathbb{Z} / d(d-2))\right)
$$

where $\rho(\mathbb{Z} / d(d-2) \mathbb{Z})=<\operatorname{diag}\left(1, \xi_{d(d-2}, \xi_{d(d-2)}^{-(d-1)}\right)>$. The automorphism groups for $d=4,6$ are given explicitly in $\S 3.3$, Proposition 15.

(4) if $\sigma$ has order $d^{2}-3 d+3$ then $\delta$ is $K$-isomorphic to the Klein curve $K_{d}: X^{d-1} Y+Y^{d-1} Z+Z^{d-1} X=0$ and for $d \geq 5$ we have $H_{K_{d}}:=\operatorname{Aut}(\delta)=<\sigma, \tau \mid \sigma^{d^{2}-3 d+3}=\tau^{3}=1$ and $\sigma \tau=\tau \sigma^{-(d-1)}>$. The locus $\left.M_{g}^{P l}\left(\mathbb{Z} /\left(d^{2}-3 d+3\right) \mathbb{Z}\right)\right)$ is irreducible with one element, and

$$
\widetilde{M_{g}\left(H_{K_{d}}\right)}=M_{g}^{P l}\left(\mathbb{Z} /\left(d^{2}-3 d+3\right)\right)=\rho\left(M_{g}^{P l}\left(\mathbb{Z} /\left(d^{2}-3 d+3\right)\right)\right)
$$

where $\rho\left(\mathbb{Z} /\left(d^{2}-3 d+3\right) \mathbb{Z}\right)=<\operatorname{diag}\left(1, \xi_{d^{2}-3 d+3}, \xi_{d^{2}-3 d+3}^{-(d-2)}\right)>$. We refer to Remark 18 of $\S 3.4$ for the classical case $d=4$.

Remark 2. The above situations do not fit with curves that have large automorphism group in the classical definition. For example, the curve $\delta: X^{d}+Y^{d-1} Z+X Z^{d-1}=0$ is defined over $\mathbb{Q}, \delta /$ Aut $(\delta)$ is a projective line and the morphism $\delta \rightarrow \delta / A u t(\delta)$ is ramified at two points of ramification index $(d-1)^{2}$ and at $d-1$-points of 
ramification index $d-1$. Therefore this curve has no a large automorphism group in any of the classical sense because it ramifies at more than 4 points. But it has "very large" elements in its automorphism group.

Now assuming that $m$ is "large" in the sense that $m \in\{\ell d, \ell(d-1), \ell(d-2): \ell \geq 2\}$, we obtain different results in $\S 4$ and $\S 5$, some of them are listed below:

Theorem 3. Let $\delta \in M_{g}^{P l}$ be a non-singular plane curve of degree $d \geq 4$ that admits an automorphism $\sigma \in \operatorname{Aut}(\delta)$ of "large" order. Then

(1) if $\sigma$ has order $\ell(d-1)$ with $\ell \geq 2$, we always have $d \equiv 0$ or $1(\bmod \ell)$ and Aut $(\delta)$ is cyclic of order $\ell^{\prime}(d-1)$ with $\ell \mid \ell^{\prime}$. If $\ell=1$, the same conclusion holds if $\sigma$ is a homology (By a homology we mean that $P^{-1} \rho(\sigma) P=\operatorname{diag}\left(1, \xi_{m}^{a}, \xi_{m}^{b}\right)$ with $a=0$ or $b=0$ for some $\left.P \in P G L_{3}(K)\right)$.

(2) if $\sigma$ has order $\ell d$ with $\ell \geq 3$ then $d \equiv 1$ or $2(\bmod \ell)$, Aut $(\delta)$ fixes a line and a point off that line (in particular, following the same notations of $\S 3$, it is an exterior group as in Theorem 9 (2) with $N$ of order $d)$. When $\ell=2$, Aut $(\delta)$ could also be conjugate to a subgroup of Aut $\left(F_{d}\right)$ where $F_{d}$ is the Fermat curve $X^{d}+Y^{d}+Z^{d}=0$ (in such cases we say that $\delta$ is a descendent of the Fermat curve, see the precise definition in $\S 3)$.

(3) if $\sigma$ has order $\ell(d-2)$ with $\ell \geq 2$ then always $d \equiv 0(\bmod \ell)$ and, roughly speaking, for $d>6$ and $d \neq 10$, we can think about Aut $(\delta)$ in a short exact sequence $1 \rightarrow \mathbb{Z} / k \rightarrow$ Aut $(\delta) \rightarrow D \rightarrow 1$ with $k$ divides $d$ and $D$ is the Dihedral group $D_{2(d-2)}$ or $D_{d-2}$. For more accurate details, we refer to $\S 4.2$

Remark 4. In the above situations where $m$ is "large", we also obtain that every element in $M_{g}^{P l}(\mathbb{Z} / m)$ is given by a certain specialization of the parameters in a fixed normal form for the full locus $M_{g}^{P l}(\mathbb{Z} / m)$. This phenomena is not true in general for an arbitrary $m$. In other words, with the aid of the algorithm in $\S 2$, we prove that $\rho\left(M_{g}^{P l}(\mathbb{Z} / m)\right)$ has the property of being represented by an unique fixed normal form. But the moduli $M_{g}^{P l}(\mathbb{Z} / m)$ with $m$ not "large" or "very large" is not in general given by a single equation with some parameters (counter examples are provided in [1]).

Remark 5. Take $K=\mathbb{C}$. Then, one should expect to have non-singular plane curves which have a "large" element in the automorphism group and no plane model (up to $\mathbb{C}$-isomorphism) defined over the algebraic closure of $\mathbb{Q}$ inside $\mathbb{C}$. Let us reproduce the situation that has been mentioned in [1, §2.1] for $d=5$ and a "large" element of order 8, as an explicit example of the above phenomena. Any element in $M_{6}^{P l}(\mathbb{Z} / 8)$ has, up to $K$-isomorphism, a plane models of the form $X^{5}+Y^{4} Z+X Z^{4}+\beta X^{3} Z^{2}=0$ for certain/s $\beta$ (note that $\beta \neq \pm 2$ for non-singularity). We constructed in [1] a bijection map

$$
\begin{gathered}
\varphi: M_{6}^{P l}(\mathbb{Z} / 8 \mathbb{Z}) \rightarrow \mathbb{A}^{1}(K) \backslash\{-2,2\} / \sim \\
\alpha \mapsto[\beta]=\{\beta,-\beta\}
\end{gathered}
$$

where $a \sim b \Leftrightarrow b=a$ or $a=-b$, and we know that the non-singular plane model $X^{5}+Y^{4} Z+X Z^{4}+\beta X^{3} Z^{2}=0$ has a bigger automorphism group than $\mathbb{Z} / 8 \mathbb{Z}$ if and only if $\beta=0$.

\section{Cyclic automorphism group of NON-Singular Plane CURVES}

Fix and integer $d \geq 4$, and consider $\delta \in M_{g}^{P l}$ such that the group $G \cong A u t(\delta)$ is non-trivial. Let $C$ : $F(X ; Y ; Z)=0$ in $\mathbb{P}^{2}(K)$ be a non-singular plane model of degree $d$ over an algebraically closed field $K$ of characteristic zero. Suppose that $\operatorname{Aut}(C)=\rho(G) \leq P G L_{3}(K)$ for some $\rho: G \hookrightarrow P G L_{3}(K)$ (any other plane model of $\delta$ is given by $C_{P}: F(P(X ; Y ; Z))=0$ for some $P \in P G L_{3}(K)$ moreover $\operatorname{Aut}\left(C_{P}\right)$ is conjugate through $P$ to $\operatorname{Aut}(C)$, and we say that $C_{P}$ is $K$-equivalent or $K$-isomorphic to $\left.C\right)$. Assume that $\rho(\sigma) \in \operatorname{Aut}(C)$ is an element of order $m$ hence by a change of variables in $\mathbb{P}^{2}$ (in particular, changing the plane model to a $K$-equivalent one associated to $\delta)$, we can consider $\rho(\sigma)$ as the automorphism $(x: y: z) \mapsto\left(x: \xi_{m}^{a} y: \xi_{m}^{b} z\right)$ where $\xi_{m}$ is a primitive $m$-th root of unity in $K$ and $a, b$ are integers such that $0 \leq a \neq b \leq m-1$. Moreover, if $a b \neq 0$ then $m$ and $\operatorname{gcd}(a, b)$ are coprime (we can reduce to $\operatorname{gcd}(a, b)=1)$ and if $a=0$ then $\operatorname{gcd}(b, m)=1$. Also, such an automorphism is identified with type $m,(a, b)$ and we write $\rho_{a, b, m}(\mathbb{Z} / m \mathbb{Z})$ for the subgroup given 


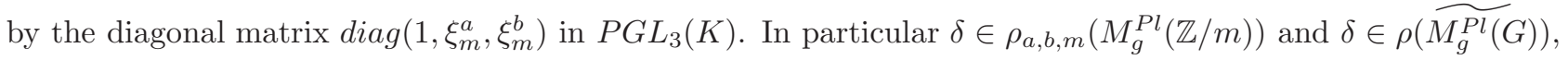
of course $\rho_{a, b, m}$ may be interpreted as the restriction to $\langle\sigma\rangle$ of $\rho$.

Our aim here is to investigate which cyclic groups could appear inside $A u t(\delta)$, thus to determine all possible types $m,(a, b)$ for which the moduli $\rho_{a, b, m}\left(M_{g}^{P l}(\mathbb{Z} / m)\right)$ might be non-empty. We follow a similar approach as Dolgachev in [5] which deal with the same question for $d=4$ (see also [3, §2.1]).

Throughout this paper, we use the following notations.

- Type $m,(a, b)$ is identified with the corresponding automorphism $\left[X ; \zeta_{m}^{a} Y ; \zeta_{m}^{b} Y\right]$ where $\zeta_{m}$ is a primitive $m$-th root of unity. Saying that $m,(a, b)$ is a generator of $\rho(\mathbb{Z} / m)$ for certain $\rho: \mathbb{Z} / m \hookrightarrow P G L_{3}(K)$ means that any element of $\rho(\mathbb{Z} / m)$ is a power of the associated automorphism to Type $m,(a, b)$.

- $L_{i, *}$ denotes a degree $i$, homogeneous polynomial in $K[X, Y, Z]$ such that the variable $* \in\{X, Y, Z\}$ does not appear.

- $S(u)_{m}:=\{j: u \leq j \leq d-1, d-j=0(\bmod m)\}$.

- $S_{u}^{d, X} m,(a, b):=\{i: u \leq i \leq d-u$ and $a i+(d-i) b=0(\bmod m)\}$.

- $S_{u}^{d-1, X} m,(a, b):=\{i: 1 \leq i \leq d-u$ and $a i+(d-1-i) b=0(\bmod m)\}$

- $S(1)_{m,(a, b)}^{j, X}:=\{i: 0 \leq i \leq j$ and $a i+(j-i) b=a(\bmod m)\}$.

- $S(2)_{m,(a, b)}^{j, X}:=\{i: 0 \leq i \leq j$ and $a i+(j-i) b=0(\bmod m)\}$.

- $S_{m,(a, b)}^{j, Y}:=\{i: 0 \leq i \leq j$ and $b i+(d-j) a=a(\bmod m)\}$.

- $S_{m,(a, b)}^{j, Z}:=\{i: 0 \leq i \leq j$ and $a i+(d-j) b=a(\bmod m)\}$.

- $\Gamma_{m}:=\left\{(a, b) \in \mathbb{N}^{2}: \operatorname{g.c.d}(a, b)=1,1 \leq a \neq b \leq m-1\right\}$.

- the points $P_{1}:=(1: 0: 0), P_{2}:=(0: 1: 0)$ and $P_{3}:=(0: 0: 1)$ inside $\mathbb{P}^{2}(K)$ are called the reference points.

- $\alpha \in K^{*}$ and it can always be 1 by a change of variables.

where $u, j, m, d, a$ and $b$ are all non-negative integers.

Theorem 6. Let $\delta \in M_{g}^{P l}$ be a non-singular projective plane curve of degree $d \geq 4$ over an algebraically closed field $K$ of zero characteristic. If $H$ is a non-trivial cyclic subgroup of Aut $(\delta)$ of order $m$, then $\delta \in$ $\rho_{a, b, m}\left(M_{g}^{P l}(\mathbb{Z} / m)\right)$ for the following list (1)-(6) of values of $a, b, m$. We associate to each locus a normal form, that is unique up to $K$-equivalence. Any plane model in $\mathbb{P}^{2}(K)$ of an element $\delta \in \rho_{a, b, m}\left(M_{g}^{P l}(\mathbb{Z} / m)\right)$ is obtained by a certain specialization of the parameters in the normal form and, any specialization of the parameters (under certain restrictions in the parameters) gives a plane non-singular model of an element of this locus: a

(1) The curve $\delta \in \rho_{m, 0,1}\left(M_{g}^{P l}(\mathbb{Z} / m)\right)$ with $m \mid d-1$ and a plane model of the curve is of the form

$$
Z^{d-1} L_{1, Z}+\left(\sum_{j \in S(2)_{m}} Z^{d-j} L_{j, Z}\right)+L_{d, Z} .
$$

(2) The curve $\delta \in \rho_{m, 0,1}\left(M_{g}^{P l}(\mathbb{Z} / m)\right)$ with $m \mid d$ and a plane model of the curve has the form

$$
Z^{d}+\left(\sum_{j \in S(1)_{m}} Z^{d-j} L_{j, Z}\right)+L_{d, Z} .
$$

(3) All reference points lie on $\delta$ : The curve $\delta \in \rho_{m, a, b}\left(M_{g}^{P l}(\mathbb{Z} / m)\right)$ with $m \mid\left(d^{2}-3 d+3\right)$ and $(a, b) \in \Gamma_{m}$ such that $a=(d-1) a+b=(d-1) b(\bmod m)$. In particular $\delta$ has a plane non-singular model where all reference points lie on it, and a plane non-singular model of $\delta$ is given by certain specialization of $\alpha, \beta_{j, i}, \alpha_{i, j}, \gamma_{i, j} \in K$ of the equation

$$
\begin{aligned}
X^{d-1} Y & +Y^{d-1} Z+\alpha Z^{d-1} X+ \\
& +\sum_{j=2}^{\left\lfloor\frac{d}{2}\right\rfloor} X^{d-j}\left(\sum_{i \in S(1)_{m,(a, b)}^{j, X}} \beta_{j, i} Y^{i} Z^{j-i}\right)+Y^{d-j}\left(\sum_{i \in S_{m,(a, b)}^{j, Y}} \alpha_{j, i} Z^{i} X^{j-i}\right)+Z^{d-j}\left(\sum_{i \in S_{m,(a, b)}^{j, Z}} \gamma_{j, i} X^{j-i} Y^{i}\right),
\end{aligned}
$$

\footnotetext{
${ }^{a}$ We warn the reader because it may happen that a projective equation, which is obtained by a certain type $m(a, b)$, is not geometrically irreducible or not non-singular for any specialization of the parameters. Hence, $\rho_{a, b, m}\left(M_{g}^{P l}(\mathbb{Z} / m)\right)$ is the empty set and then should be discarded from the list.
} 
(4) Two reference points lie on $\delta$ : One of the following subcases occurs.

(4.1) $\delta \in \rho_{m, a, b}\left(M_{g}^{P l}(\mathbb{Z} / m)\right)$ where $m \mid d(d-2)$ and $(a, b) \in \Gamma_{m}$ such that $(d-1) a+b \equiv 0(\bmod m)$ and $a+(d-1) b \equiv 0(\bmod m)$. Moreover, a plane model $C$ of $\delta$ is given by a certain specialization of the parameters of the equation

$$
X^{d}+\left(\sum_{j=2}^{d-1} X^{d-j} \sum_{i \in S(2)_{m,(a, b)}^{j, X}} \beta_{j, i} Y^{i} Z^{j-i}\right)+\left(Y^{d-1} Z+\alpha Y Z^{d-1}+\sum_{i \in S_{2}^{d, X}{ }_{m,(a, b)}} \beta_{d, i} Y^{i} Z^{d-i}\right)=0
$$

(4.2) $\delta \in \rho_{m, a, b}\left(M_{g}^{P l}(\mathbb{Z} / m)\right)$ where $m \mid(d-1)^{2}$ and $(a, b) \in \Gamma_{m}$ such that $(d-1) a+b \equiv 0(\bmod m)$ and $(d-1) b \equiv 0(\bmod m)$. Furthermore, a plane non-singular model $C$ of $\delta$ is obtained by a certain specialization of the parameters of the equation

$$
\begin{aligned}
X^{d} & +\sum_{j=2}^{d-2} X^{d-j}\left(\sum_{i \in S(2)_{m,(a, b)}^{j, X}} \beta_{j, i} Y^{i} Z^{j-i}\right)+X\left(\alpha Z^{d-1}+\sum_{i \in S_{1}^{d-1, X}} \beta_{m,(a, b)} \beta_{(d-1), i} Y^{i} Z^{d-1-i}\right)+ \\
& +\left(Y^{d-1} Z+\sum_{i \in S_{2}^{d, X}{ }_{m,(a, b)}} \beta_{d, i} Y^{i} Z^{d-i}\right)=0
\end{aligned}
$$

(4.3) $\delta \in \rho_{m, a, b}\left(M_{g}^{P l}(\mathbb{Z} / m)\right)$ where $m \mid(d-1)$ and $(a, b) \in \Gamma_{m}$ such that $(d-1) b \equiv 0(\bmod m)$ and $(d-1) a \equiv 0(\bmod m)$. In such case a plane non-singular model $C$ of $\delta$ has the form

$$
\begin{aligned}
X^{d} & +\sum_{j=2}^{d-2}\left(X^{d-j} \sum_{i \in S(2)_{m,(a, b)}^{j, X}} \beta_{j, i} Y^{i} Z^{j-i}\right)+\sum_{i \in S_{2}^{d, X} m,(a, b)} \beta_{d, i} Y^{i} Z^{d-i}+ \\
& +X\left(Z^{d-1}+\alpha Y^{d-1}+\sum_{i \in S_{2}^{d-1, X}} \beta_{(d-1), i} Y^{i} Z^{d-1-i}\right),
\end{aligned}
$$

(5) One reference points lie on $\delta$ : Then $\delta \in \rho_{m, a, b}\left(M_{g}^{P l}(\mathbb{Z} / m)\right)$ with $m \mid d(d-1)$ and $(a, b) \in \Gamma_{m}$ such that $d a \equiv 0(\bmod m)$ and $(d-1) b \equiv 0(\bmod m)$. Also, a plane model of $\delta$ is given by the form

$$
\begin{aligned}
X^{d} & +Y^{d}+\sum_{j=2}^{d-2}\left(X^{d-j} \sum_{i \in S(2)_{m,(a, b)}^{j, X}} \beta_{j, i} Y^{i} Z^{j-i}\right)+\sum_{i \in S_{1}^{d, X}} \beta_{m,(a, b)} \beta_{d, i} Y^{i} Z^{d-i}+ \\
& +X\left(\alpha Z^{d-1}+\sum_{i \in S_{1}^{d-1, X}} \beta_{(d-1), i} Y^{i} Z^{d-1-i}\right)=0
\end{aligned}
$$

(6) None of the reference points lie on a plane model $C$ of $\delta$, then $\delta \in \rho_{m, a, b}\left(M_{g}^{P l}(\mathbb{Z} / m)\right)$ where $m \mid d$ and $(a, b) \in \Gamma_{m}$ such that $d a \equiv 0(\bmod m)$ and $d b \equiv 0(\bmod m)$. Furthermore, we have

$$
X^{d}+Y^{d}+Z^{d}+\sum_{j=2}^{d-1}\left(X^{d-j} \sum_{i \in S(2)_{m,(a, b)}^{j, X}} \beta_{j, i} Y^{i} Z^{j-i}\right)+\sum_{i \in S_{1}^{d, X}} \beta_{m,(a, b)} Y^{i} Z^{d-i}=0 .
$$

Here, $\alpha, \beta_{i, j}, \gamma_{i, j}, \alpha_{i, j}$ are parameters which specialize, for a concrete $\delta$ as above, at values in $K$ with always $\alpha \neq 0$.

Remark 7. The above result and its proof give an algorithm to list, for every fixed degree d, all cyclic groups that could appear with an equation (up to $K$-isomorphism). For the complete algorithm and its implementation in SAGE, see the link http://mat.uab.cat/ eslam/CAGPC.sagews. Also see the appendix for a list of Types that could appear for degree $d \leq 9$ (i.e. the possible non-trivial $\rho_{m, a, b}\left(M_{g}^{P l}(\mathbb{Z} / m)\right.$ ) loci for a fixed degree $d \leq 9$ ) with their equations that are given by parameters. These equations assign to specializations of the parameters, plane models of the elements of the loci $\rho_{m, a, b}\left(M_{g}^{P l}(\mathbb{Z} / m)\right)$.

Proof. Without loss of generality, we consider a plane model $C: F(X ; Y ; Z)=0$ of $\delta$ such that the cyclic element order $m$ acts as the diagonal matrix $\operatorname{diag}\left(1, \xi_{m}^{a}, \xi_{m}^{b}\right)$ in the plane equation $F(X ; Y ; Z)=0$. Let $\varphi$ be a generator of order $m:=|H|$. One can choose coordinates so that $\varphi$ is represented by $(x ; y, z) \mapsto\left(x ; \xi_{m}^{a} y, \xi_{m}^{b} z\right)$ where $a, b$ are integers with $0 \leq a \neq b \leq m-1$ ( one can assume that $a<b$ with $\operatorname{gcd}(b, m)=1$ if $a=0$ and 
with $\operatorname{gcd}(a, b)=1$ otherwise):

Case I : Suppose first that $a=0$ and write: $F(X ; Y ; Z)=\lambda Z^{d}+\left(\sum_{j=1}^{d-1} Z^{d-j} L_{j, Z}\right)+L_{d, Z}$.

If $\lambda=0$, then by non-singularity $L_{1, Z} \neq 0$ and $(d-1) b=0(\bmod m)$. Hence, $m \mid d-1$ and we can take a generator $(a, b)=(0,1)$. Therefore, by checking each monomial's invariance, we obtain that $L_{j, Z} \neq 0$ only if $j \in S(2)_{m}$ and we get types $m,(0,1)$ of $(1)$.

If $\lambda \neq 0$ then $d b \equiv 0(\bmod m)$. From which we obtain $m \mid d$ and $(a, b)=(0,1)$ is a generator for each such $m$. By the same discussion as before, we have types $m,(0,1)$ of the form $Z^{d}+\left(\sum_{j \in S(1)_{m}} Z^{d-j} L_{j, Z}\right)+L_{d, Z}$, which proves (2).

Case II : Suppose that $a \neq 0$ then necessarily, $m>2$ and we distinguish between the following four subcases:

i.: All reference points lie in $C$,

ii.: Two reference points lie in $C$,

iii.: One reference point lies in $C$,

iv.: None of the reference points lie in $C$.

- If all reference points lie on $C$, then the possibilities for the defining equation are now:

$$
C: \sum_{j=1}^{\left\lfloor\frac{d}{2}\right\rfloor}\left(X^{d-j} L_{j, X}+Y^{d-j} L_{j, Y}+Z^{d-j} L_{j, Z}\right)
$$

Because $a \neq b$ with $a \neq 0$, we can assume that $C: X^{d-1} Y+Y^{d-1} Z+\alpha Z^{d-1} X+\sum_{j=2}^{\left\lfloor\frac{d}{2}\right\rfloor}\left(X^{d-j} L_{j, X}+\right.$ $\left.Y^{d-j} L_{j, Y}+Z^{d-j} L_{j, Z}\right)$. The first three factors implies that $a \equiv(d-1) a+b \equiv(d-1) b(\bmod m)$. In particular, $m \mid d^{2}-3 d+3$. The defining equation (3) follows immediately by checking monomials' invariance in each $L_{j, B}$. For example, rewrite $L_{j, X}$ as $\sum_{i=0}^{j} \beta_{j, i} Y^{i} Z^{j-i}$ then $\beta_{j, i}=0$ if $m \nmid a i+(j-i) b$ or equivalently $i \notin S(1)_{m,(a, b)}^{j, X}$, since $\operatorname{diag}\left(1 ; \xi_{m}^{a} ; \xi_{m}^{b}\right) \in \operatorname{Aut}(C) b$

- If two reference points lie on $C$, then by re-scaling the matrix $\varphi$ and permuting the coordinates, we can assume that $(1 ; 0 ; 0) \notin C$. The equation is then $C: X^{d}+X^{d-2} L_{2, X}+X^{d-3} L_{3, X}+\ldots+X L_{d-1, X}+L_{d, X}=0$, since $L_{1, X}$ is not invariant by $\varphi$ because $a b \neq 0$. Moreover, $Z^{d}$ and $Y^{d}$ are not in $L_{d, X}$, by the assumption that only $(1 ; 0 ; 0) \notin C$. Assume first that $Y^{d-1} Z$ and $Y Z^{d-1}$ are in $L_{d, X}$. Then $(d-1) a+b \equiv 0(\bmod m)$ and $a+(d-1) b \equiv 0(\bmod m)$. In particular, $m \mid d(d-2)$ and for each such type $m,(a, b)$, the equation is reduced to $X^{d}+\left(\sum_{j=2}^{d-1} X^{d-j} \sum_{i=0}^{j} \beta_{j, i} Y^{i} Z^{j-i}\right)+\left(Y^{d-1} Z+\alpha Y Z^{d-1}+\sum_{i=2}^{d-2} \beta_{d, i} Y^{i} Z^{d-i}\right)=0$. It is straightforward to see that if $i \notin S(2)_{m,(a, b)}^{j, X}\left(\right.$ resp. $\left.i \notin S_{2}^{d, X} m,(a, b)\right)$ then $\beta_{j, i}=0$ (resp. $\left.\beta_{d i}=0\right)$. This proves (4.1). Secondly, assume that $Y^{d-1} Z \in L_{d, X}$ and $Y Z^{d-1} \notin L_{d, X}$. Then, by the non-singularity, $Z^{d-1}$ is in $L_{d-1, X}$. That is $(d-1) a+b \equiv 0(\bmod m)$ and $(d-1) b \equiv 0(\bmod m)$. Therefore $m \mid(d-1)^{2}$ and we have the form

$$
X^{d}+\alpha X Z^{d-1}+Y^{d-1} Z+\sum_{j=2}^{d-2} \sum_{i=0}^{j} \beta_{j, i} X^{d-j} Y^{i} Z^{j-i}+\sum_{i=1}^{d-1} \beta_{(d-1), i} X Y^{i} Z^{d-1-i}+\sum_{i=2}^{d-2} \beta_{d, i} Y^{i} Z^{d-i}=0 .
$$

Consequently, by checking the monomials' invariance, we conclude that if $i \notin S(2)_{m,(a, b)}^{j, X}$ then $\beta_{j, i}=0$, if $i \notin S_{1}^{d-1, X} m,(a, b)$ then $\beta_{(d-1), i}=0$, if $i \notin S_{2}^{d, X} m,(a, b)$ then $\beta_{d, i}=0$ and the result follows for (4.2). Up to a permutation of $Y$ and $Z$, it remains to consider the case for which $Y^{d-1} Z$ and $Y Z^{d-1}$ are not in $L_{d, X}$. By the non-singularity, $Z^{d-1}$ and $Y^{d-1}$ should be in $L_{d-1, X}$ consequently, $(d-1) b \equiv 0(\bmod m)$ and $(d-1) a \equiv 0(\bmod m)$. Therefore, $m \mid(d-1)$ and the form is reduced to

$$
X^{d}+X Z^{d-1}+\alpha X Y^{d-1}+\sum_{j=2}^{d-2} \sum_{i=0}^{j} \beta_{j, i} X^{d-j} Y^{i} Z^{j-i}+\sum_{i=2}^{d-2} \beta_{d, i} Y^{i} Z^{d-i}+\sum_{i=1}^{d-2} \beta_{(d-1), i} X Y^{i} Z^{d-1-i}=0,
$$

and the equation (4.3) is now clear by the fact that $\beta_{j, i}=0$ whenever $m \nmid a i+(j-i) b$.

\footnotetext{
${ }^{b}$ It is to be noted that for a fixed $m$ and $\left(a_{0}, b_{0}\right) \in L_{m}$ where $L_{m}:=\left\{(a, b) \in \Gamma_{m}: a \equiv(d-1) a+b \equiv(d-1) b(\bmod m)\right\}$, the type $m,\left(a_{0}, b_{0}\right)$ is $K$-isomorphic to any type $m,\left(a^{\prime}, b^{\prime}\right) \in<m,(a, b)>$. So, to complete the classification for $m$, it suffices to choose another $(a, b) \in L_{m}-<\left(a_{0}, b_{0}\right)>$ and repeat until we get $L_{m}=\emptyset$.
} 
- If one reference points lie in the $C$, then by normalizing the matrix $\varphi$ and permuting the coordinates, we can assume that $(1 ; 0 ; 0),(0 ; 1 ; 0) \notin C$. We then write

$$
C: X^{d}+Y^{d}+X^{d-2} L_{2, X}+X^{d-3} L_{3, X}+\ldots+X L_{d-1, X}+L_{d, X}=0
$$

such that $Z^{d} \notin L_{d, X}$. Also, by the non-singularity, we have $Z^{d-1} \in L_{d-1, X}$. In particular, $d a \equiv$ $0(\bmod m)$ and $(d-1) b \equiv 0(\bmod m)$ and $m \mid d(d-1)$. The above equation become

$$
X^{d}+Y^{d}+\alpha X Z^{d-1}+\sum_{j=2}^{d-2} \sum_{i=0}^{j} \beta_{j, i} X^{d-j} Y^{i} Z^{j-i}+\sum_{i=1}^{d-1} \beta_{d, i} Y^{i} Z^{d-i}+\sum_{i=1}^{d-1} \beta_{(d-1), i} X Y^{i} Z^{d-1-i}=0
$$

Following the same line of argument as before, we conclude (5).

- If none of the reference points lie in $C$ then $C: X^{d}+Y^{d}+Z^{d}+\left(\sum_{j=2}^{d-1} X^{d-j} L_{j, X}\right)+L_{d, X}=0$, where $L_{1, X}$ does not appear since $a b \neq 0$ and $L_{1, X}$ is not invariant under $\varphi$. Clearly $d a \equiv d b \equiv 0(\bmod m)$ and therefore $m \mid d$. Moreover

$$
C: X^{d}+Y^{d}+Z^{d}+\sum_{j=2}^{d-1} \sum_{i \in S(2)_{m,(a, b)}^{j, X}} \beta_{j, i} X^{d-j} Y^{i} Z^{j-i}+\sum_{i \in S_{1}^{d, X}} \beta_{m,(a, b)} Y_{d i}^{i} Z^{d-i}=0 .
$$

This completes the proof of our result.

Corollary 8. Let $H$ be a non-trivial cyclic subgroup of Aut $(\delta)$ where $\delta \in M_{g}^{P l}$ with $d \geq 4$. Then the order of $H$ divides one of the integers $d-1, d, d^{2}-3 d+3,(d-1)^{2}, d(d-2), d(d-1)$. Consequently automorphisms of $\delta$ have orders $\leq d(d-1)$.

\section{Characterization of Curves $\delta \in M_{g}^{P l}$ Whose $A u t(\delta)$ has "Very large" Elements}

We study here non-singular plane curves $\delta \in M_{g}^{P l}$ that admits a $\sigma \in A u t(\delta)$ of "very large" or "large" order: $d^{2}-3 d+3,(d-1)^{2}, d(d-2), d(d-1), \ell(d-1)$ or $\ell d$ with $\ell \geq 2$. In particular we are interested in investigating the full automorphism group and the corresponding non-singular plane equations (up to $K$-isomorphism) of such curves.

Before a detailed study of the automorphism groups for such $\delta$ 's, we recall the following general results concerning $A u t(\delta)$ for $\delta \in M_{g}^{P l}$ which will be useful throughout this paper. In some cases we will use the notation of the GAP library for small finite groups to indicate some of them.

Because linear systems $g_{d}^{2}$ are unique (up to multiplication by $P \in P G L_{3}(K)$ in $\mathbb{P}^{2}(K)$ [10, Lemma 11.28]), we always consider a non-singular plane model $C$ of $\delta$, which is given by a projective plane equation $F(X ; Y ; Z)=0$ and $A u t(C)$ is a finite subgroup of $P G L_{3}(K)$ that fixes the equation $F$ and is isomorphic to $A u t(\delta)$. Any other plane model of $\delta$ is given by $C_{P}: F(P(X ; Y ; Z))=0$ with $A u t\left(C_{P}\right)=P^{-1} A u t(C) P$ for some $P \in P G L_{3}(K)$ and $C_{P}$ is $K$-equivalent or $K$-isomorphic to $C$. By an abuse of notation, we also denote a non-singular projective plane curve of degree $d$ by $C$. Therefore, $A u t(C)$ satisfies one of the following situations (see Mitchel [13] for more details):

(1) fixes a point $Q$ and a line $L$ with $Q \notin L$ in $P G L_{3}(K)$,

(2) fixes a triangle (i.e. a set of three non-concurrent lines),

(3) Aut $(C)$ is conjugate to a representation inside $P G L_{3}(K)$ of one of the finite primitive group namely, the Klein group $P S L(2,7)$, the icosahedral group $A_{5}$, the alternating group $A_{6}$, the Hessian group $H_{e s s_{216}}$ or to one of its subgroups $\mathrm{Hess}_{72}$ or $\mathrm{Hess}_{36}$.

It is classically known that if a subgroup $H$ of automorphisms of a non-singular plane curve $C$ fixes a point on $C$ then $H$ is cyclic [10, Lemma 11.44], and recently Harui in [8, §2] provided the lacked result in the literature on the type of groups that could appear for non-singular plane curves. Before introducing the statement of Harui, we need to define the terminology of being a descendent of a plane curve. For a non-zero monomial $c X^{i} Y^{j} Z^{k}$ with $c \in K \backslash\{0\}$ we define its exponent as $\max \{i, j, k\}$. For a homogenous polynomial $F$, the core of $F$ is defined to be the sum of all terms of $F$ with the greatest exponent. Let $C_{0}$ be a smooth plane curve, a pair $(C, H)$ with $H \leq A u t(C)$ is said to be a descendant of $C_{0}$ if $C$ is defined by a homogenous polynomial whose core is a defining polynomial of $C_{0}$ and $H$ acts on $C_{0}$ under a suitable change of the coordinate system. 
Theorem 9 (Harui). If $H \preceq A u t(C)$ where $C$ is a non-singular plane curve of degree $d \geq 4$ then $H$ satisfies one of the following.

(1) $H$ fixes a point on $C$ and then cyclic.

(2) $H$ fixes a point not lying on $C$ and satisfies a short exact sequence of the form $1 \rightarrow N \rightarrow H \rightarrow G^{\prime} \rightarrow 1$, where $N$ a cyclic group of order dividing $d$ and $G^{\prime}$ (which is a subgroup of $P G L_{2}(K)$ ) is conjugate to a cyclic group $\mathbb{Z} / m \mathbb{Z}$ of order $m$ with $m \leq d-1$, a Dihedral group $D_{2 m}$ of order $2 m$ where $|N|=1$ or $m \mid(d-2)$, the alternating groups $A_{4}, A_{5}$ or the symmetry group $S_{4}$.

(3) $H$ is conjugate to a subgroup of $A$ ut $\left(F_{d}\right)$ where $F_{d}$ is the Fermat curve $X^{d}+Y^{d}+Z^{d}$. In particular, $|H| \mid 6 d^{2}$ and $(C, H)$ is a descendant of $F_{d}$.

(4) $H$ is conjugate to a subgroup of Aut $\left(K_{d}\right)$ where $K_{d}$ is the Klein curve curve $X Y^{d-1}+Y Z^{d-1}+Z X^{d-1}$ hence $|H| \mid 3\left(d^{2}-3 d+3\right)$ and $(C, H)$ is a descendant of $K_{d}$.

(5) $\mathrm{H}$ is conjugate to a finite primitive subgroup of $P G L_{3}(K)$ that are mentioned above.

Now assume, as usual, that $C$ is a non-singular plane model of degree $d \geq 4$ with $\sigma \in A u t(C)$ of exact order $m$ that acts on $F(X ; Y ; Z)=0$ as $(x, y, z) \mapsto\left(x, \xi_{m}^{a} y, \xi_{m}^{b} z\right)$. In the next sections, mainly in the proofs, we recall the abuse of notation of refereing to $C$ as a non-singular plane curve (up to $K$-isomorphism) instead of being a non-singular plane model of some $\delta \in M_{g}^{P l}$.

3.1. The locus $M_{g}^{P l}(\mathbb{Z} /(d(d-1)))$.

The following result appears in Harui [8, §3].

Proposition 10 (Harui). For any $\left.\left.d \geq 5, \delta \in M_{g}^{P l}(\widetilde{\mathbb{Z} /(d(d}-1)\right)\right)$ if and only if $\delta$ has a plane model given by $C: X^{d}+Y^{d}+X Z^{d-1}=0$.

Moreover we prove the following:

Proposition 11. For $d \geq 4, \delta \in M_{g}^{P l}(\mathbb{Z} / d(d-1))$ if and only if $\delta$ has a non-singular plane model that is $K$-equivalent to $C: X^{d}+Y^{d}+\alpha X Z^{d-1}=0$ where $\alpha \neq 0$ (always we can assume $\alpha=1$ by a K-isomorphic model to $C)$. Consequently, $M_{g}^{P l}(\mathbb{Z} / d(d-1) \mathbb{Z})$ is an irreducible locus with one element. Furthermore, for $d \geq 5$,

$$
\left.M_{g}^{P l}(\widetilde{\mathbb{Z} / d(d}-1)\right)=M_{g}^{P l}(\mathbb{Z} / d(d-1))=\rho\left(M_{g}^{P l}(\mathbb{Z} / d(d-1))\right)
$$

where $\rho(\mathbb{Z} / d(d-1) \mathbb{Z})=<\operatorname{diag}\left(1, \xi_{d(d-1)}^{d-1}, \xi_{d(d-1)}^{d}\right)>$.

Remark 12. Recall that for $d=4$, the automorphism group of $X^{4}+Y^{4}+\alpha X Z^{3}=0$ is isomorphic to $\mathbb{Z} / 4 \odot A_{4}$, the element of $\operatorname{Ext}^{1}\left(A_{4}, \mathbb{Z} / 4\right) \subseteq$ which is given by $\left\{(\delta, g) \in \mu_{12} \times H: \delta^{4}=\chi(g)\right\} / \pm 1$, where $\mu_{n}$ is the group of $n$-th roots of unity, $H$ is the group $A_{4}$ and $\chi$ is the character $\chi: H \rightarrow \mu_{3}$ defined by $\chi(S)=1$ and $\chi(T)=\rho$ where $S, T$ are generators of $H$ of order 2 and 3 respectively with the representation $H=<S, T \mid S^{2}=1, T^{3}=1, \ldots>$ and $\rho$ is a 3rd-primitive root of unity, see [9] (or also [3]).

Proof. If $\delta$ has a non-singular plane model which is isomorphic to $C: X^{d}+Y^{d}+\alpha X Z^{d-1}=0$ then $\delta \in$ $M_{g}^{P l}(\mathbb{Z} / d(d-1))$ because $\left[X ; \zeta_{d(d-1)}^{d-1} Y ; \zeta_{d(d-1)}^{d} Z\right]$ is an element of $A u t(C)$ of order $d(d-1)$. Conversely, suppose that $\delta \in M_{g}^{P l}(\mathbb{Z} / d(d-1))$ and fix as usual, by an abuse of notation, a plane non-singular model $C$ (up to $K$-isomorphism) of $\delta$. Since $d(d-1)$ does not divide any of the integers $d-1, d, d^{2}-3 d+3, d(d-2),(d-1)^{2}$ then by Theorem 6. $C$ is projectively equivalent to type $d(d-1),(a, b)$ of the form (5) for some $(a, b) \in \Gamma_{d(d-1)}$ such that $d a \equiv 0 \bmod d(d-1)$ and $(d-1) b \equiv 0 \bmod d(d-1)$. In particular $a=(d-1) k$ and $b=d k^{\prime}$ for some integers $k$ and $k^{\prime}$ and since we have $\left[X ; \zeta_{d(d-1)}^{d-1} Y ; \zeta_{d(d-1)}^{d} Z\right]^{d\left(k^{\prime}-k\right)+k}=\left[X ; \zeta_{d(d-1)}^{(d-1) k} Y ; \zeta_{d(d-1)}^{d k^{\prime}} Z\right]$ then $m,(a, b)$ with $m=d(d-1), a=d-1$ and $b=d$ is a generator of such types. Hence

${ }^{c}$ We use the notation $\operatorname{Ext}^{1}(A, B)$ to represent the group $G$ (up to isomorphism) for which there is an exact sequence of groups of the form $1 \rightarrow B \rightarrow G \rightarrow A \rightarrow 1$. 


$$
\begin{aligned}
S(2)_{m,(a, b)}^{j, X}: & =\{i: 0 \leq i \leq j \text { and }(d-1) i+(j-i) d=0 \bmod d(d-1)\} \\
& =\{i: 0 \leq i \leq j \text { and } d(d-1) \mid(d j-i)\} \\
& =\emptyset \forall j=2, \ldots, d-2 \text { (because } 0<d j-i<d(d-1)),
\end{aligned}
$$

Also

$$
\begin{aligned}
S_{1}^{d, X} & :=\{i: 1 \leq i \leq d-1 \text { and }(d-1) i+(d-i) d=0 \bmod d(d-1)\} \\
& =\{i: 1 \leq i \leq d-1 \text { and } d(d-1) \mid d-i\} \\
& =\emptyset \text { because } 0<d-i<d(d-1)), \\
S_{1}^{d-1, X} & :=\{i: 1 \leq i \leq d-1 \text { and }(d-1) i+(d-1-i) d=0 \bmod d(d-1)\} \\
& =\{i: 1 \leq i \leq d-1 \text { and } d(d-1) \mid i\} \\
& =\emptyset .
\end{aligned}
$$

Therefore, by substituting in the form (5) of Theorem 6. $C$ is isomorphic to $X^{d}+Y^{d}+\alpha X Z^{d-1}$ where $\alpha \neq 0$. The last part is an immediate consequence of Proposition 10 .

3.2. The moduli $M_{g}^{P l}\left(\mathbb{Z} /(d-1)^{2}\right)$.

Proposition 13. For any $d \geq 4$, if $\delta \in M_{g}^{P l}$ has a non-singular plane model that is isomorphic to

$$
C: X^{d}+Y^{d-1} Z+\alpha X Z^{d-1}=0
$$

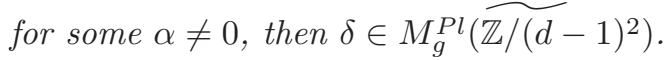

Proof. The result is is well-known for $d=4$ (see [9] or 3] for more details), so we assume that $d \geq 5$. We have $\left[X ; \zeta_{d-1} Y ; Z\right] \in A u t(C)$ which is a homology of order $d-1 \geq 4$ hence $A u t(C)$ should fix a point, a line or a triangle ( see $\S 5$ of Mitchell [13]). Since $\left[X ; \zeta_{(d-1)^{2}} Y ; \zeta_{(d-1)^{2}}^{(d-1)(d-2)} Z\right] \in A u t(C)$ is of order $(d-1)^{2}$ then also $(d-1)^{2}|| A u t(C) \mid$. Now assume that $A u t(C)$ fixes a triangle and neither a line nor a point is fixed by $A u t(C)$ then it follows by the proof of Theorem 9 (see [8, §4]), that $C$ is either a descendent of the Fermat curve $F_{d}$ or the Klein curve $K_{d}$. But, none of these curves admits automorphisms of order $(d-1)^{2}$, since elements of $A u t\left(F_{d}\right)$ (resp. Aut $\left(K_{d}\right)$ ) have orders at most $2 d$ (resp. $d^{2}-3 d+3$ ). Secondly, if $A u t(C)$ fixes a point not lying on $C$ then we can think about $A u t(C)$ in a short exact sequence $1 \rightarrow N \rightarrow A u t(C) \rightarrow G^{\prime} \rightarrow 1$ as in Theorem 9 (2). Since $|N|$ and $(d-1)^{2}$ are coprime, then $(d-1)^{2} \| G^{\prime} \mid$ which is not possible for any of the groups $\mathbb{Z} / m, A_{4}, S_{4}, A_{5}$ or $D_{2 m}$ with $m \leq d-1$. Consequently, $A u t(C)$ fixes a point on $C$ and hence it is cyclic of order divisible by $(d-1)^{2}$ and $\leq d(d-1)$. That is, $\operatorname{Aut}(C)$ is cyclic of order $(d-1)^{2}$. In particular $\delta \in M_{g}^{P l}\left(\widetilde{\mathbb{Z} /(d-1)^{2}}\right)$.

The following is an analogue of Proposition 11 .

Proposition 14. For $d \geq 4, \delta \in M_{g}^{P l}\left(\mathbb{Z} /(d-1)^{2}\right)$ if and only if $\delta$ has a non-singular plane model which is isomorphic to $C: X^{d}+Y^{d-1} Z+\alpha X Z^{d-1}=0$ with $\alpha \neq 0$. Therefore $M_{g}^{P l}\left(\mathbb{Z} /(d-1)^{2} \mathbb{Z}\right)$ is an irreducible locus with one element and $\left.M_{g}^{P l}(\widetilde{\mathbb{Z} / d(d}-1)\right)=M_{g}^{P l}(\mathbb{Z} / d(d-1))=\rho\left(M_{g}^{P l}(\mathbb{Z} / d(d-1))\right)$ where $\rho(\mathbb{Z} / d(d-1) \mathbb{Z})$ is $<\operatorname{diag}\left(1, \xi_{(d-1)^{2}}, \xi_{(d-1)^{2}}^{(d-1)(d-2)}\right)>$. Furthermore, if $G$ is a non-cyclic automorphism group of a non-singular plane curve and $(d-1)^{2}|| G \mid$ then $G$ does not contain any element of such order.

Proof. We only need to show that $\delta \in M_{g}^{P l}\left(\mathbb{Z} /(d-1)^{2}\right)$ only if $\delta$ has a non-singular plane model that is isomorphic to $C: X^{d}+Y^{d-1} Z+\alpha X Z^{d-1}=0$ with $\alpha \neq 0$, since the remaining parts are immediate consequences of Proposition 13 Up to projective equivalence, we consider a model $C$ of $\delta$ in $\rho\left(M_{g}^{P l}(\mathbb{Z} / d(d-1))\right)$ and since $(d-1)^{2} \nmid d-1, d, d^{2}-3 d+3, d(d-2), d(d-1)$ then $C$ is isomorphic to type $(d-1)^{2},(a, b)$ of the form (4.2) of Theorem [6. In particular $(a, b) \in \Gamma_{(d-1)^{2}}$ such that $(d-1) a+b \equiv 0 \bmod (d-1)^{2},(d-1) b \equiv 0 \bmod (d-1)^{2}$ and $a=(d-1) k-k^{\prime}, b=(d-1) k^{\prime}$ for some integers $k$ and $k^{\prime}$. But we have

$$
\left[X ; \zeta_{(d-1)^{2}} Y ; \zeta_{(d-1)^{2}}^{(d-1)(d-2)} Z\right]^{(d-1) k-k^{\prime}}=\left[X ; \zeta_{(d-1)^{2}}^{(d-1) k-k^{\prime}} Y ; \zeta_{(d-1)^{2}}^{(d-1) k^{\prime}} Z\right]
$$


That is $m=(d-1)^{2}, a=1$ and $b=(d-1)(d-2)$ is a generator of such types. Moreover

$$
\begin{aligned}
S(2)^{j, X} & :=\left\{i: 0 \leq i \leq j \text { and } i+(j-i)(d-1)(d-2)=0 \bmod (d-1)^{2}\right\} \\
& =\left\{i: 0 \leq i \leq j \text { and }(d-1)^{2} \mid j(d-1)-d i\right\} \\
& =\emptyset \forall j=2, \ldots, d-2 .
\end{aligned}
$$

The last equality follows because $(d-1)^{2} \mid j(d-1)-d i$ implies that $d-1 \mid i$ thus $i=0$. But then we must have $(d-1)^{2} \mid j(d-1)$ which is impossible since $0<j<d-1$. Also, we have

$$
\begin{aligned}
S_{2}^{d, X} & :=\left\{i: 2 \leq i \leq d-2 \text { and } i+(d-i)(d-1)(d-2)=0 \bmod (d-1)^{2}\right\} \\
& \subseteq\{i: 2 \leq i \leq d-2 \text { and } d-1 \mid i\} \\
& =\emptyset \\
S_{1}^{d-1, X} & :=\left\{i: 1 \leq i \leq d-1 \text { and } i+(d-1-i)(d-1)(d-2)=0 \bmod (d-1)^{2}\right\} \\
& =\left\{i: 1 \leq i \leq d-1 \text { and }(d-1)^{2} \mid d i\right\} \\
& =\emptyset .
\end{aligned}
$$

Substituting into equation (4.2) yields that $C$ is isomorphic to the equation $X^{d}+Y^{d-1} Z+\alpha X Z^{d-1}=0$ and we are done.

3.3. The moduli $M_{g}^{P l}(\mathbb{Z} / d(d-2))$. Assume that $\delta \in M_{g}^{P l}$ has a non-singular plane model isomorphic to the curve $C: X^{d}+Y^{d-1} Z+\alpha Y Z^{d-1}=0$ of degree $d \geq 4$. The full automorphism group of $\delta$ is given by the following result:

Proposition 15. Consider $\delta \in M_{g}^{P l}$ with the above property. Therefore Aut $(\delta)$ is is the central extension $<\sigma, \tau \mid \sigma^{2}=\tau^{d(d-2)}=1$ and $\sigma \tau \sigma=\tau^{-(d-1)}>$ of $D_{2(d-2)}$ by $\mathbb{Z} / d$ whenever $d \neq 4,6$. In particular Aut $(\delta)$ is of order $2 d(d-2)$. For $d=6$, it is a central extension of $S_{4}$ by $\mathbb{Z} / 6$ thus $|A u t(C)|=144$ and for $d=4$, $\delta$ is isomorphic to the Fermat quartic curve $F_{4}$ hence $\operatorname{Aut}(\delta) \simeq(\mathbb{Z} / 4)^{2} \rtimes S_{3}$.

Proof. Let $\mu \in K$ such that $\mu^{d(d-2)}=\alpha$, then $C$ is projectively equivalent, through the transformation $\left[X ; \mu Y ; \mu^{-(d-1)} Z\right]$, to the curve $C^{\prime}: X^{d}+Y^{d-1} Z+Y Z^{d-1}=0$ and hence it follows, by $\S 6$ of Harui [8], that $A u t\left(C^{\prime}\right)$ is isomorphic to $\mathbb{Z}_{4}^{2} \rtimes S_{3}$ (for $d=4$ ), a central extension of $S_{4}$ by $\mathbb{Z} / d$ (for $d=6$ ) and a central extension of $D_{2(d-2)}$ by $\mathbb{Z} / d(d \neq 4,6)$. Finally, it is to be noted that $\sigma:=[X ; Z ; Y]$ and $\tau:=\left[X ; \zeta_{d(d-2)} Y ; \zeta_{d(d-2)}^{-(d-1)} Z\right]$ generate $\operatorname{Aut}\left(C^{\prime}\right)$ for $d \neq 4,6$ which completes the proof.

Similarly to Propositions 11 and 14, we prove:

Proposition 16. A curve $\delta$ of $d \geq 4$ belongs to $M_{g}^{P l}(\mathbb{Z} / d(d-2))$ if and only if it has plane models that are isomorphic to $C: X^{d}+Y^{d-1} Z+Y Z^{d-1}=0$. Hence $M_{g}^{P l}(\mathbb{Z} / d(d-2))$ is irreducible and consists of a single element. Furthermore $\left.\widehat{M_{g}^{P l}\left(H_{d}\right.}\right)=M_{g}^{P l}(\mathbb{Z} / d(d-1))=\rho\left(M_{g}^{P l}(\mathbb{Z} / d(d-1))\right)$ where $H_{d}$ is the concrete central extension of $D_{2(d-2)}$ by $\mathbb{Z} / d(d \neq 4,6)$, a central extension of $S_{4}$ by $\mathbb{Z} / d(d=6)$ or $\simeq(\mathbb{Z} / 4)^{2} \rtimes S_{3}(d=4)$, which are detailed in Proposition 15 , and $\rho(\mathbb{Z} / d(d-2) \mathbb{Z})=<\operatorname{diag}\left(1, \xi_{d(d-2)}, \xi_{d(d-2)}^{-(d-1)}\right)>$.

Proof. It suffices to prove the "only if" statement since otherwise is straightforward by Proposition 15 have by Theorem 6 and because $d(d-2) \nmid d-1, d, d^{2}-3 d+3,(d-1)^{2}, d(d-1)$ that any plane model of $\delta$ is isomorphic to type $d(d-2),(a, b)$ of the form (4.1) of Theorem 6 . That is $(a, b) \in \Gamma_{d(d-2)}$ such that $(d-1) a+b \equiv 0 \bmod d(d-2)$ and $a+(d-1) b \equiv 0 \bmod d(d-2)$. In particular, $a=k$ and $b=d k^{\prime}+k$ for some integers $k$ and $k^{\prime}$ such that $k$ and $d k^{\prime}+k$ are coprime and $d-2 \mid k+k^{\prime}$. Consequently we can take a generator 
$k=1$ and $k^{\prime}=d-3$, since $\left[X ; \zeta_{d(d-2)} Y ; \zeta_{d(d-2)}^{d(d-3)+1} Z\right]^{k}=\left[X ; \zeta_{d(d-2)}^{k} Y ; \zeta_{d(d-2)}^{d k^{\prime}+k} Z\right]$. Therefore

$$
\begin{aligned}
S(2)^{j, X} & :=\{i: 0 \leq i \leq j \text { and } i+(j-i)(d(d-3)+1)=0 \bmod d(d-2)\} \\
& =\{i: \quad 0 \leq i \leq j \text { and } d(d-2) \mid j(d-1)-d i\} \\
& =\emptyset \forall j=2, \ldots, d-2 \text { (because if } d(d-2) \mid j(d-1)-d i \text { then } d \mid j \text { a contradiction) } \\
S_{2}^{d, X} & :=\{i: 2 \leq i \leq d-2 \text { and } i+(d-i)(d(d-3)+1)=0 \bmod d(d-2)\} \\
& \subseteq\{i: 2 \leq i \leq d-2 \text { and } d-2 \mid d-1-i\} \\
& =\emptyset .
\end{aligned}
$$

Hence $C$ is isomorphic to $X^{d}+Y^{d-1} Z+\alpha Y Z^{d-1}$ with $\alpha \neq 0$.

3.4. The moduli $M_{g}^{P l}\left(\mathbb{Z} /\left(d^{2}-3 d+3\right)\right.$. The next result is well-known in the literature, see for example [8, §3].

Proposition 17. If $\delta \in M_{g}^{P l}$ has a non-singular plane model of degree $d \geq 5$ that is $K$ - equivalent to

$$
C: X^{d-1} Y+Y^{d-1} Z+\alpha Z^{d-1} X=0,
$$

where $\alpha \neq 0$. Then Aut $(\delta)$ is isomorphic to $<\tau, \sigma \mid \tau^{d^{2}-3 d+3}=\sigma^{3}=1$ and $\tau \sigma=\sigma \tau^{-(d-1)}>$, a semidirect product of $\mathbb{Z} / 3$ by $\mathbb{Z} /\left(d^{2}-3 d+3\right)$ and hence $A u t(\delta)$ is of order $3\left(d^{2}-3 d+3\right)$.

Proof. Through the transformation $\left[X ; \mu Y ; \mu^{-(d-2)} Z\right]$ where $\mu$ is defined by the equation $\alpha=\mu^{d^{2}-3 d+3}$ in $K$, $C$ is isomorphic to the Klein curve $K_{d}$. It follows, by Harui $[8] \S 3$, that $\operatorname{Aut}(C)$ is a semidirect product of $\mathbb{Z} / 3$ acting on $\mathbb{Z} /\left(d^{2}-3 d+3\right)$. Finally we note that $\tau:=\left[X ; \zeta_{d^{2}-3 d+3} Y ; \zeta_{d^{2}-3 d+3}^{-(d-2)} Z\right]$ and $[Z ; X ; Y]$ are generators of $\operatorname{Aut}\left(K_{d}\right)$ and also satisfy the given representation.

Remark 18. The automorphism group of the Klein quartic curve is isomorphic to $P S L_{2}\left(\mathbb{F}_{7}\right)$, the unique simple group of order 168 (see [9]). This completes the result for any degree $d \geq 4$.

The following result should be well-known in the literature, we write it for completeness.

Proposition 19. We have $\delta \in M_{g}^{P l}\left(\mathbb{Z} /\left(d^{2}-3 d+3\right)\right)$ only if $\delta$ is isomorphic to the Klein curve

$$
K_{d}: X^{d-1} Y+Y^{d-1} Z+Z^{d-1} X=0 .
$$

In particular, $M_{g}^{P l}\left(\mathbb{Z} /\left(d^{2}-3 d+3\right)\right)$ is irreducible being a set with one element and also

$$
\left.M_{g}^{P l} \widetilde{(\operatorname{Aut}}\left(K_{d}\right)\right)=M_{g}^{P l}\left(\mathbb{Z} /\left(d^{2}-3 d+3\right)\right)=\rho\left(M_{g}^{P l}\left(\mathbb{Z} /\left(d^{2}-3 d+3\right)\right)\right)
$$

where $\rho\left(\mathbb{Z} / d^{2}-3 d+3 \mathbb{Z}\right)=<\operatorname{diag}\left(1, \xi_{d^{2}-3 d+3}, \xi_{d^{2}-3 d+3}^{-(d-2)}\right)>$.

Proof. Since $d^{2}-3 d+3 \nmid d-1, d, d(d-1), d(d-2),(d-1)^{2}$ for every $d \geq 5$ then $C$ is $K$-equivalent to a plane curve of type $d^{2}-3 d+3,(a, b)$ of the form (3) in Theorem 6 for some $(a, b) \in \Gamma_{d^{2}-3 d+3}$ such that $a=(d-1) a+b=$ $(d-1) b\left(\bmod d^{2}-3 d+3\right)$. In particular every solution is of the form $a=k$ and $b=\left(d^{2}-3 d+3\right) k^{\prime}-(d-2) k$ for some integers $k$ and $k^{\prime}$. Because $\left[X ; \zeta_{d^{2}-3 d+3} Y ; \zeta_{d^{2}-3 d+3}^{d^{2}-4 d+5}\right]^{k}=\left[X ; \zeta_{d^{2}-3 d+3}^{k} Y ; \zeta_{d^{2}-3 d+3}^{\left(d^{2}-3 d+3\right) k^{\prime}-(d-2) k}\right]$, we can take a generator $a=1$ and $b=d^{2}-4 d+5$. Consequently

$$
\begin{aligned}
S(1)^{j, X} & :=\left\{i: 0 \leq i \leq j \text { and } i+(j-i)\left(d^{2}-4 d+5\right)=1 \bmod \left(d^{2}-3 d+3\right)\right\} \\
& =\left\{i: 0 \leq i \leq j \text { and }\left(d^{2}-3 d+3\right) \mid(j(d-2)-i(d-1)+1)\right\} \\
& =\emptyset \quad \forall j=2, \ldots,\left\lfloor\frac{d}{2}\right\rfloor .
\end{aligned}
$$

The last equality comes from the fact $|j(d-2)-i(d-1)+1|<d^{2}-3 d+3$ then $j(d-2)-i(d-1)+1=0$. This in turns gives $d \mid 2 j-i-1$ which is impossible because $0<2 j-i-1<d$. Also

$$
\begin{aligned}
S^{j, Z} & :=\left\{i: \quad 0 \leq i \leq j \text { and } i+(d-j)\left(d^{2}-4 d+5\right)=1 \bmod \left(d^{2}-3 d+3\right)\right\} \\
& =\left\{i: \quad 0 \leq i \leq j \text { and }\left(d^{2}-3 d+3\right) \mid((d-j)(d-2)-i+1)\right\} \\
& =\emptyset \forall j=2, \ldots,\left\lfloor\frac{d}{2}\right\rfloor\left(\text { since } 0<(d-j)(d-2)-i+1<d^{2}-3 d+3\right)
\end{aligned}
$$


Moreover

$$
\begin{aligned}
S^{j, Y} & :=\left\{i: 0 \leq i \leq j \text { and }\left(d^{2}-4 d+5\right) i+(d-j)=1 \bmod \left(d^{2}-3 d+3\right)\right\} \\
& =\left\{i: 0 \leq i \leq j \text { and }\left(d^{2}-3 d+3\right) \mid((d-j)-(d-2) i-1)\right\} \\
& =\emptyset \quad \forall j=2, \ldots,\left\lfloor\frac{d}{2}\right\rfloor,
\end{aligned}
$$

since $|(d-j)-(d-2) i-1|<d^{2}-3 d+3$ and if $(d-j)-(d-2) i-1=0$ then $d-2 \mid j-1$ a contradiction because always $0<j-1<d-2$. Therefore $C$ is isomorphic to $X^{d-1} Y+Y^{d-1} Z+\alpha Z^{d-1} X$ with $\alpha \neq 0$. The full automorphism of the Klein curve is classified by Proposition 17 and the second statement is proved.

\section{Characterization of Curves $\delta \in M_{g}^{P l}$ Whose $A u t(\delta)$ has "Large" elements}

In the previous section we proved that if $m$ is "very large", the moduli $M_{g}^{P l}(\mathbb{Z} / m)$ is given by one element, therefore are irreducible set. In general it is difficult, for an arbitrary $m$, to decide whether the set $M_{g}^{P l}(\mathbb{Z} / m)$ is irreducible or not. We introduced in [1] a weaker concept than irreducibility that we call "ES-irreducibility", where a loci $\rho\left(M_{g}^{P l}(G)\right)$ or $M_{g}^{P l}(G)$ is said to be $E S$-irreducible if it is defined, up to $K$-isomorphism of plane curves, via a single projective equation of degree $d$ together with certain parameters that are associated to the equation under some algebraic constraints, in other words, by an unique normal form up to $K$-isomorphism. Also any element of the locus corresponds to a specific specialization of the parameters and vice versa. In particular the "very large"- $m$ loci $M_{g}^{P l}(\mathbb{Z} / m)$ that appeared in $\S 2$ are ES-irreducible. It is not true in general that $M_{g}^{P l}(\mathbb{Z} / m)$ is ES-irreducible, see counter examples in [1], and therefore is not irreducible as a subset of the moduli space $M_{g}$.

We show here that a "large"- $m$ locus $M_{g}^{P l}(\mathbb{Z} / m)$ is ES-irreducible and we obtain further details of such loci. The situations where $m \in\{\ell d, \ell(d-1)\}$ are strongly related to inner and outer Galois points (we refer to [15] for more details) which will help in determining, more precisely, the automorphism groups of these loci in some cases.

One can read Henn [9] or [3] for the well-known results in the literature on quartic curves. Hence, in what follows, we assume that $d \geq 5$.

\subsection{Outer and inner Galois points with $d \geq 5$.}

We are interested in non-singular plane curves $\delta \in M_{g}^{P l}$ of an arbitrary but a fixed degree $d \geq 5$ whose automorphism groups contain homologies of period $d$ (resp. $d-1$ ). Recall that a homology is a finite planar transformation such that by a change of variables it is the same as certain type $m,(a, b)$ with $a b=0$ (see Mitchell [13]). When a homology $\omega$ of period $d$ or $d-1$ is present inside $A u t(\delta)$, the genus of $\delta /\langle\omega\rangle$ is zero and $\delta$ has a unique outer (resp. inner) Galois point $P$ (see [8, Lemma 3.7] for existence and [15] for the definition of an inner or an outer Galois point as well as the uniqueness in such cases $d$ ). Furthermore if a non-singular plane curve $\delta$ of degree $d \geq 5$ has an outer (resp. an inner) Galois point $P$, then $\tau(P)$ is also an outer (resp. an inner) Galois point of $\delta$ for any $\tau \in A u t(\delta)$. Consequently if $\delta$ has an unique inner Galois point then it should be fixed by the full automorphism group $A u t(\delta)$ hence by [10, Lemma 11.44], Aut $(\delta)$ is a cyclic group provided that $\operatorname{Char}(K)=0$.

4.1.1. The loci $M_{g}^{P l}(\mathbb{Z} / \ell(d-1))$ with $2 \leq \ell \leq d$.

Lemma 20. The locus $M_{g}^{P l}(\mathbb{Z} / \ell(d-1))$ where $2 \leq \ell \leq d$ is not empty only if $d \equiv 0(\bmod \ell)$ or $d \equiv 1(\bmod \ell)$.

Proof. Since $\ell(d-1) \nmid d-1, d, d^{2}-3 d+3, d(d-2)$ then $\ell(d-1) \mid d(d-1)$ or $(d-1)^{2}$ by Corollary 8

\footnotetext{
${ }^{d}$ An outer Galois point, if it exists, is always unique except when the curve is isomorphic to the Fermat curve, in such case there are exactly 3 outer Galois points.
} 
Proposition 21. Assume that $d \geq 5$ and $2 \leq \ell \leq d$ with $d \equiv 0(\bmod \ell)$, then $\delta \in M_{g}^{P l}(\mathbb{Z} / \ell(d-1))$ if and only if $\delta$ has a non-singular plane model that is $K$-isomorphic to

$$
C: X^{d}+Y^{d}+\alpha X Z^{d-1}+\sum_{2 \leq \ell k \leq d-2} \beta_{\ell k, \ell k} X^{d-\ell k} Y^{\ell k},
$$

In particular Aut $(\delta)$ is a cyclic group of order divisible by $\ell(d-1)$.

Proof. $(\Leftarrow)$ Since $\sigma:=\left[X ; \zeta_{\ell(d-1)}^{d-1} Y ; \zeta_{\ell(d-1)}^{\ell} Z\right] \in A u t(C)$ is of order $\ell(d-1)$ then $\delta \in M_{g}^{P l}(\mathbb{Z} / \ell(d-1))$ and moreover $C$ is not a descendant of the Klein curve $K_{d}$ because $\ell(d-1) \nmid 3\left(d^{2}-3 d+3\right)$. Also $C$ is not a descendant of the Fermat curve $F_{d}$, since $2(d-1) \nmid 6 d^{2}$ and $\ell(d-1)>2 d$ for $\ell \geq 3$ but Aut $\left(F_{d}\right)$ has elements of order at most $2 d$. On the other hand, $\sigma^{\ell}=\left[X ; Y ; \zeta_{\ell(d-1)}^{\ell^{2}} Z\right] \in A u t(C)$ is a homology of period $d-1 \geq 4$ with center $P_{3}$ and axis $Z=0$. Therefore the point $P_{3}$ is an inner Galois point of $C$ (by Harui [8, §3]) and it is unique (by Yoshihara [15, §2, Theorem 4']) hence should be fixed by $\operatorname{Aut}(C)$. Consequently $\operatorname{Aut}(C)$ is a cyclic group of order divisible by $\ell(d-1)$.

$\Leftrightarrow$ Conversely, $\ell(d-1) \nmid d-1, d, d^{2}-3 d+3,(d-1)^{2}$ or $d(d-2)$ therefore $\delta$ has a non-singular plane model which is isomorphic to type $\ell(d-1),(a, b)$ of the form (5) of Theorem 6 In particular $(a, b) \in \Gamma_{\ell(d-1)}$ such that $\ell(d-1) \mid d a$ and $\ell(d-1) \mid(d-1) b$ therefore $a=(d-1) k$ and $b=\ell k^{\prime}$ for some integers $k$ and $k^{\prime}$. If we consider any integer $m$ such that $k \equiv m(\bmod \ell)$ then $\left[X ; \zeta_{\ell(d-1)}^{d-1} Y ; \zeta_{\ell(d-1)}^{\ell} Z\right]^{\left(k^{\prime}-m\right)(d-1)+k^{\prime}}=\left[X ; \zeta_{\ell(d-1)}^{k(d-1)} Y ; \zeta_{\ell(d-1)}^{\ell k^{\prime}} Z\right]$. Consequently we can take $k=1=k^{\prime}$ as a generator and we get

$$
\begin{aligned}
S_{1}^{d, X} & :=\{i: 1 \leq i \leq d-1 \text { and }(d-1) i+(d-i) \ell=0 \bmod \ell(d-1)\} \\
& =\{i: 1 \leq i \leq d-1 \text { and } \ell(d-1) \mid(d-1) i-(i-1) \ell\} \\
& \subseteq\{i: 1 \leq i \leq d-1 \text { and }(d-1) \mid(i-1)\}=\{1\} .
\end{aligned}
$$

Since $\ell(d-1) \nmid(d-1)(\ell+1)$ then $S_{1}^{d, X}=\emptyset$. Also

$$
\begin{aligned}
S_{1}^{d-1, X} & :=\{i: 1 \leq i \leq d-1 \text { and }(d-1) i+(d-1-i) \ell=0 \bmod \ell(d-1)\} \\
& \subseteq\{i: 1 \leq i \leq d-1 \text { and }(d-1) \mid i\}=\{d-1\} .
\end{aligned}
$$

But $\ell(d-1) \nmid(d-1)^{2}$ by the hypothesis on $\ell$, therefore $S_{1}^{d-1, X}=\emptyset$. Moreover

$$
\begin{aligned}
S(2)^{j, X}: & =\{i: 0 \leq i \leq j \text { and }(d-1) i+(j-i) \ell=0 \bmod \ell(d-1)\} \\
& \subseteq\{i: 0 \leq i \leq j \text { and }(d-1) \mid j-i\}=\{j\}(\text { since } 0 \leq j-i<d-1)
\end{aligned}
$$

By assumption, $\sigma \in A u t(\delta)$ therefore $S(2)_{m,(a, b)}^{j, X}=\emptyset$ if $\ell \nmid j$ and $\{j\}$ otherwise. Substituting into equation (5) in Theorem [6], we obtain the defining equation (1).

We also obtain a similar result when $d \equiv 1(\bmod \ell)$ :

Proposition 22. Assume that $d \geq 5$ and $2 \leq \ell \leq d$ with $d \equiv 1(\bmod \ell)$, then $\delta \in M_{g}^{P l}(\mathbb{Z} / \ell(d-1))$ if and only if $\delta$ has a non-singular plane model that is $K$-isomorphic to

$$
X^{d}+Y^{d-1} Z+\alpha X Z^{d-1}+\sum_{2 \leq \ell k \leq d-2} \beta_{\ell k, 0} X^{d-\ell k} Z^{\ell k}
$$

In such case, Aut $(\delta)$ is again cyclic of order divisible by $\ell(d-1)$.

Proof. $(\Leftarrow)$ We need only to redefine $\sigma$ to be the automorphism $\left[X ; \zeta_{\ell(d-1)} Y ; \zeta_{\ell(d-1)}^{(\ell-1)(d-1)} Z\right]$ and the rest of the argument will be quite similar.

$(\Rightarrow)$ It follows by Corollary 8 that $\delta$ has a non-singular plane model which is isomorphic to type $\ell(d-1),(a, b)$ of the form (4.2) of Theorem [6. In particular $(a, b) \in \Gamma_{\ell(d-1)}$ such that $\ell(d-1) \mid(d-1) a+b,(d-1) b$ therefore $b=$ $(d-1) k^{\prime}$ and $a=\ell k-k^{\prime}$ for some integers $k$ and $k^{\prime}$. But $\left[X ; \zeta_{\ell(d-1)} Y ; \zeta_{\ell(d-1)}^{(\ell-1)(d-1)} Z\right]^{\ell k-k^{\prime}}=\left[X ; \zeta_{\ell(d-1)}^{a} Y ; \zeta_{\ell(d-1)}^{b} Z\right]$ 
therefore it suffices to consider $k=1$ and $k^{\prime}=\ell-1$ and we obtain

$$
\begin{aligned}
& S_{1}^{d-1, X}:=\{i:1 \leq i \leq d-1 \text { and } i+(d-1-i)(\ell-1)(d-1)=0 \bmod \ell(d-1)\} \\
&=\{i: 1 \leq i \leq d-1 \text { and } \ell(d-1) \mid d i\}=\emptyset(\text { because } 0<i<\ell(d-1)), \\
& S_{2}^{d, X}:=\{i: \quad 2 \leq i \leq d-2 \text { and } i+(d-i)(\ell-1)(d-1)=0 \bmod \ell(d-1)\} \\
&=\{i: \quad 2 \leq i \leq d-2 \text { and } \ell(d-1) \mid d i-(d-1)\} \\
& \subseteq\{i: \quad 2 \leq i \leq d-2 \text { and } d-1 \mid d i\}=\emptyset(\text { because } 0<i<d-1), \\
& S(2)^{j, X}:=\{i: 0 \leq i \leq j \text { and } i+(j-i)(\ell-1)(d-1)=0 \bmod \ell(d-1)\} \\
&=\{i: 0 \leq i \leq j \text { and } \ell(d-1) \mid d i-j(d-1)\} \\
& \subseteq\{i: 0 \leq i \leq j \text { and } d-1 \mid d i\}=\{0\} .
\end{aligned}
$$

But $\ell(d-1) \mid j(d-1)$ whenever $i=0$ thus $\ell \mid j$. Therefore equation (2) is obtained by substituting in the form (4.2) of Theorem 6 .

The following corollaries are immediate consequences of Propositions 21 and 22

Corollary 23. The loci $M_{g}^{P l}(\mathbb{Z} / \ell(d-1))$ with $2 \leq \ell \leq d$ and $d \geq 5$ are empty or ES-irreducible given by one normal form.

Corollary 24. The automorphism group of any $\delta \in M_{g}^{P l}(\mathbb{Z} / \ell(d-1))$ with $2 \leq \ell \leq d$ is cyclic and always contains a homology of period $d-1$. In particular $\delta$ has a unique inner Galois point.

Remark 25. The converse of Corollary 24 is also true. In the sense that, if $C$ is a non-singular projective plane curve of degree $d \geq 5$ such that $A$ ut $(C)$ contains a homology $\sigma$ of order $d-1$ with center $P$ then $C$ has an inner Galois point $P$ by [8, Lemma 3.7] and moreover it is unique by [15, Theorem 4]. This point should be fixed by $A u t(C)$ which in turns implies that Aut $(C)$ is cyclic by [10, Lemma 11.44]. .

4.1.2. The loci $M_{g}^{P l}(\mathbb{Z} / \ell d)$ with $2 \leq \ell \leq d-1$.

Lemma 26. The locus $M_{g}^{P l}(\mathbb{Z} / \ell d)$ where $2 \leq \ell \leq d-1$ is not empty only if $d=1(\bmod \ell)$ or $d \equiv 2(\bmod \ell)$.

Proof. The result follows by Corollary 8 , since $\ell d \nmid d-1, d, d^{2}-3 d+3,(d-1)^{2}$.

Proposition 27. Assume that $d \geq 5$ and $3 \leq \ell \leq d-1$ with $d \equiv 1(\bmod \ell)$, then $\delta \in M_{g}^{P l}(\mathbb{Z} / \ell d)$ if and only if $\delta$ has a non-singular plane model that is $K$-isomorphic to

$$
\tilde{C}: X^{d}+Y^{d}+\alpha X Z^{d-1}+\sum_{2 \leq \ell k \leq d-2} \beta_{\ell k, 0} X^{d-\ell k} Z^{\ell k}
$$

where $\alpha \neq 0$. In this case, Aut $(\delta)$ should fix a line and a point off that line and every automorphism of $\delta$ is projectively equivalent to a transformation of the form $\left[\alpha_{1} X+\alpha_{3} Z ; Y ; \gamma_{1} X+\gamma_{3} Z\right]$.

Proof. $(\Leftarrow)$ Since $\sigma:=\left[X ; \zeta_{\ell d}^{\ell} Y ; \zeta_{\ell d}^{d} Z\right] \in A u t(\tilde{C})$ is of order $\ell d$ then $\delta \in M_{g}^{P l}(\mathbb{Z} / \ell d)$ and moreover $\sigma^{\ell} \in A u t(\tilde{C})$ is a homology of period $d>4$ with center $P_{2}$ and axis $Y=0$. In particular, by [13, Aut $(\tilde{C})$ fixes a line and a point off that line or it fixes a triangle. Assume that it fixes a triangle and neither a point nor line is leaved invariant, then $\tilde{C}$ is a descendant of the Klein curve $K_{d}$ or the Fermat curve $F_{d}$ which is impossible because $\ell d \nmid 3\left(d^{2}-3 d+3\right)$ and elements of $A u t\left(F_{d}\right)$ have orders at most $2 d<\ell d$. Consequently a line and a point off that line is leaved invariant. Also it follows by $[8]$, that the point $P_{2}$ is an outer Galois point of $\tilde{C}$. Moreover it is unique because $\tilde{C}$ is not isomorphic to the Fermat curve $F_{d}$ ( [15] $\S 2$ Theorem $\left.4^{\prime}\right)$ hence this point should be fixed by $\operatorname{Aut}(\tilde{C})$. Furthermore the axis $Y=0$ should also be fixed (see [13, Theorem 4) that is automorphisms of $\tilde{C}$ are of the form $\left[\alpha_{1} X+\alpha_{3} Z ; Y ; \gamma_{1} X+\gamma_{3} Z\right]$.

$(\Rightarrow)$ Conversely, one may follow the same line of argument in Proposition 21 to conclude that $\tilde{C}$ is isomorphic to type $\ell d,\left(\ell k, d k^{\prime}\right)$ of the form (5) of Theorem [6 and to figure out that we can assume $k=1=k^{\prime}$ as a generator, 
since $\left[X ; \zeta_{\ell d}^{\ell} Y ; \zeta_{\ell d}^{d} Z\right]^{\left(k^{\prime}-m\right) d+k}=\left[X ; \zeta_{\ell d}^{\ell k} Y ; \zeta_{\ell d}^{d k^{\prime}} Z\right]$ where $k \equiv m(\bmod \ell)$. In this case, we get

$$
\begin{aligned}
S_{1}^{d, X}: & =\{i: 1 \leq i \leq d-1 \text { and } \ell i+(d-i) d=0 \bmod \ell d\} \\
& =\{i: 1 \leq i \leq d-1 \text { and } \ell d \mid i(d-\ell)-d\} \\
& \subseteq\{i: 1 \leq i \leq d-1 \text { and } d \mid i\}=\emptyset .
\end{aligned}
$$

Similarly $S_{1}^{d-1, X} \subseteq\{i: 1 \leq i \leq d-1$ and $d \mid i\}=\emptyset$. Furthermore $i \in S(2)^{j, X}$ iff $\ell d \mid \ell i-(j-i) d$ thus $d \mid i$ and $i=0$. That is $i \in S(2)^{j, X} \neq \emptyset$ only if $\ell \mid j$ which completes the proof.

Remark 28. For $\ell=2$, proposition 27 is true with the same proof if we assume that $\delta$ is not a descendent of the Fermat curve of degree $d$.

There is a similar statement to the previous results when $\ell \mid d-2$. We state only the result since the proof can be obtained through similar techniques:

Proposition 29. Assume that $d \geq 5$ and $2 \leq \ell \leq d-1$ with $d \equiv 2(\bmod \ell)$, then $\delta \in M_{g}^{P l}(\mathbb{Z} / \ell d)$ if and only if $\delta$ has a non-singular plane model that is $K$-isomorphic to

$$
\widehat{C}: X^{d}+Y^{d-1} Z+\alpha Y Z^{d-1}+\sum_{2 \leq i=\ell k+1 \leq d-2} \beta_{d, i} Y^{i} Z^{d-i}=0 .
$$

Moreover $\widehat{C}$ is a descendant of the Fermat curve $F_{d}$ (only if $\ell=2$ ) or Aut $(\delta)$ fixes a line and a point off this line (in particular automorphisms of $\widehat{C}$ have the form $\left[X ; \beta_{2} Y+\beta_{3} Z ; \gamma_{2} Y+\gamma_{3} Z\right]$ ).

Remark 30. Unfortunately it may happen here that different families of groups appear as the full automorphism of $\delta \in M_{g}^{P l}(\mathbb{Z} / \ell d)$ depending on the specialization of the parameters.

Corollary 31. The loci $M_{g}^{P l}(\mathbb{Z} / \ell d)$ with $2 \leq \ell \leq d-1$ and $d \geq 5$ are empty or ES-irreducible.

It is well known by [8, Lemma 3.7] that if $A u t(\delta)$ has a homology of period $d$ then $\delta$ has an outer Galois point. Moreover if $\delta$ is isomorphic to the Fermat curve of degree $d$, then it has two more outer Galois points and it is unique otherwise [15, Theorem 4' and Proposition 5']. Furthermore we conclude the following:

Corollary 32. For any $\delta \in M_{g}^{P l}(\mathbb{Z} / \ell d)$ with $3 \leq \ell \leq d-1$, Aut $(\delta)$ always contains a homology of period $d$. In particular $\delta$ has an unique outer Galois point.

4.2. On the loci $M_{g}^{P l}(\mathbb{Z} / \ell(d-2) \mathbb{Z})$.

We investigate here the finite groups $G$ that contain cyclic subgroups of order $\ell(d-2)$ and for which the locus $M_{g}^{P l}(G)$ may be not empty. This question is completely solved when $d=4$ (see [9]) and $d=5$ (see [2]) therefore we assume in this part that $d \geq 6$ and also $\ell \geq 2$.

Lemma 33. The locus $M_{g}^{P l}(\mathbb{Z} / \ell(d-2))$ with $d \geq 6$ and $\ell \geq 2$ is non-empty only if $d \equiv 0(\bmod \ell)$.

Proof. We have $d \geq 6>2+\frac{2}{\ell-1}$ therefore $\ell(d-2)>d$ and $\ell(d-2) \nmid d-1$ or $d$. Also $(d-1)^{2}=d(d-2)+1, d(d-1)=$ $d(d-2)+d$ and $d^{2}-3 d+3=(d-1)(d-2)+1$ thus $\ell(d-2) \nmid(d-1)^{2}, d(d-1)$ or $d^{2}-3 d+3$, since $(d-2) \nmid d$ or 1 . Now the result follows by Corollary 8

We treat first the situation when $\ell$ is even:

Proposition 34. Suppose that $\ell \geq 2$ is an even integer such that $\ell \mid d$ with $d \geq 6$. Any $\delta \in M_{g}^{P l}(\mathbb{Z} / \ell(d-2) \mathbb{Z})$ has a plane non-singular model of the form

$$
X^{d}+Y^{d-1} Z+\alpha Y Z^{d-1}+\sum_{k=1}^{\left\lfloor\frac{d}{2 \ell}\right\rfloor} \beta_{2 \ell k, \ell k} X^{d-2 \ell k} Y^{\ell k} Z^{\ell k}=0
$$

In this case, the locus $M_{g}^{P l}(\mathbb{Z} / \ell(d-2) \mathbb{Z})$ is ES-irreducible. 
Proof. If $\delta \in M_{g}^{P l}(\mathbb{Z} / \ell(d-2) \mathbb{Z})$ then $\delta$ has an automorphism $\sigma$ of order $\ell(d-2)$. Consequently $\tau:=\sigma^{\frac{\ell}{2}} \in A u t(\delta)$ is of order $2(d-2)$, that is $\delta \in M_{g}^{P l}(\mathbb{Z} / 2(d-2) \mathbb{Z})$. Therefore we need only to deal with the case $\ell=2$. It follows by Lemma 33 that a non-singular plane model $C_{(a, b)}$ of $\delta$ should be isomorphic to type $2(d-2),(a, b)$ of the form (4.1) of Theorem 6 for some $(a, b) \in \Gamma_{2(d-2)}$ and $2(d-1) \mid(d-1) a+b, a+(d-1) b$. Clearly $(1, d-3) \in \Gamma_{2(d-2)}$ is a solution of this system and $\left[X ; \xi_{2(d-2)} Y ; \xi_{2(d-2)}^{d-3} Z\right] \in A u t\left(C_{(1, d-3)}\right)$. On the other hand, $2 \mid a-b$ and $d-2 \mid a+b$, in particular $a=k+\left(\frac{d-2}{2}\right) k^{\prime}$ and $b=-k+\left(\frac{d-2}{2}\right) k^{\prime}$ for some integers $k$ and $k^{\prime}$ and we get $2 \mid \pm k+\left(\frac{d}{2}\right) k^{\prime}$. Consequently $\left[X ; \xi_{2(d-2)} Y ; \xi_{2(d-2)}^{d-3} Z\right]^{k+\left(\frac{d-2}{2}\right) k^{\prime}}=\left[X ; \xi_{2(d-2)}^{a} Y ; \xi_{2(d-2)}^{b} Z\right]$ and $m=2(d-2), a=1$ and $b=d-3$ is a generator of the set of solution of our system. Furthermore the associated sets $S_{2}^{d, X}$ and $S(2)^{j, X}$ for $j=2, \ldots, d-1$ are computed as follows:

$$
\begin{aligned}
S_{2}^{d, X} & :=\{i: 2 \leq i \leq d-2 \text { and } 2(d-2) \mid i+(d-i)(d-3)\} \\
& \subseteq\{i: 2 \leq i \leq d-2 \text { and }(d-2) \mid 2(i-1)\} \\
& =\left\{\frac{d}{2}\right\}
\end{aligned}
$$

since $0<2(i-1)<2(d-2)$ therefore $2(i-1)=d-2$. Also we have

$$
\begin{aligned}
S(2)_{m,(a, b)}^{j, X} & :=\{i: 0 \leq i \leq j \text { and } 2(d-2) \mid i+(j-i)(d-3)\} \\
& \subseteq\{i: 0 \leq i \leq j \text { and }(d-2) \mid j-2 i\},
\end{aligned}
$$

But $|j-2 i| \leq d-1$ therefore $j-2 i=0$ or $\pm(d-2)$. In particular, $S(2)^{j, X}=\emptyset$ if $j$ is odd and $\left\{\frac{j}{2}, \frac{j \pm(d-2)}{2}\right\}$ if $j$ is even. Moreover $0 \leq i \leq j$ thus when $j$ is even and $<d-2, S(2)^{j, X}=\left\{\frac{j}{2}\right\}$ and when $j=d-2$, $S(2)^{d-2, X}=\left\{0, \frac{d-2}{2}, d-2\right\}$. Consequently, we obtain the form

$$
X^{d}+Y^{d-1} Z+\alpha Y Z^{d-1}+X^{2}\left(\beta_{d-2,0} Z^{d-2}+\beta_{0, d-2} Y^{d-2}\right)+\sum_{j=2,4, \ldots, d-2, d} \beta_{j, \frac{j}{2}} X^{d-j} Y^{\frac{j}{2}} Z^{\frac{j}{2}}=0
$$

Because $\left[X ; \xi_{\ell(d-2)} Y ; \xi_{\ell(d-2)}^{d-3} Z\right] \in A u t\left(C_{1, d-3}\right)$ hence $\beta_{d-2,0}=\beta_{0, d-2}=0$ moreover $\beta_{j,\left(\frac{j}{2}\right)}=0$ if $2 \ell \nmid j$. To deal $\ell>2$ even one obtain the result y impose that the automorphism associated to Type $\ell,(a, b)$ leaves invariant the equation.

Proposition 35. Let $\ell \geq 2$ be an even integer such that $\ell \mid d$ with $d \geq 6$ and let $G$ be a finite group inside $P G L_{3}(K)$. Then $\delta \in M_{g}^{P l}(\mathbb{Z} / \ell(d-2) \mathbb{Z}) \cap \widehat{M_{g}^{P l}(G)}$ only if one of the following situations occurs:

(1) $d=6$ and $G$ is conjugate to a central extension of $S_{4}$ by $\mathbb{Z} / 6 \mathbb{Z}$. In this case, $G$ is of order 144 and $\widehat{M_{g}^{P l}(G)}$ is an irreducible set that is given by one element which has a plane non-singular model of the form $X^{6}+Y^{5} Z+Y Z^{5}=0$.

(2) $d>6$ and $G$ is conjugate to $\langle\sigma, \tau| \tau^{2}=\sigma^{d(d-2)}=1, \tau \sigma \tau=\sigma^{-(d-1)}>$, a central extension of order $2 d(d-2)$ of $D_{2(d-2)}$ by $\mathbb{Z} / d \mathbb{Z}$. Also $\widehat{M_{g}^{P l}(G)}$ is an irreducible set and is given by one element with a non-singular plane model isomorphic to $X^{d}+Y^{d-1} Z+Y Z^{d-1}=0$.

(3) $d=6$ and $G$ is isomorphic to SmallGroup $(16,8)$ in GAP library. Furthermore any element of $M_{10}^{P l}(\operatorname{SmallGroup}(16,8))$ has a non-singular plane model, $K$-isomorphic, to $X^{6}+Y^{5} Z+Y Z^{5}+\beta_{4,2} X^{2} Y^{2} Z^{2}=$ 0 for certain $\beta_{4,2} \neq 0$.

(4) $d=10$ and $G$ is isomorphic to SmallGroup $(32,19)$ in GAP library. Similarly $M_{36}^{P l}($ SmallGroup $(32,19))$ consists of a curves which has a non-singular plane model (up to K-equivalence) of the form $X^{10}+Y^{9} Z+$ $Y Z^{9}+\beta_{6,4} X^{6} Y^{2} Z^{2}+\beta_{2,8} X^{2} Y^{4} Z^{4}=0$ with $\left(\beta_{6,4}, \beta_{2,8}\right) \neq(0,0)$.

(5) $d \neq 6,10$ and $G$ is an element $\operatorname{Ext}^{1}\left(N, D_{2(d-2)}\right)$ where $N$ is a cyclic group of order $2 r(\mid d)$. Moreover $G$ contains $\left\langle\sigma, \tau: \tau^{2}=\sigma^{\ell(d-2)}=1\right.$ and $\tau \sigma \tau=\sigma^{-(d-1)}>$ as a subgroup. Also every element of $\widehat{M_{g}^{P l}(G)}$ has a non-singular plane model of the form (5) of Proposition 34 such that $\beta_{2 k \ell, \ell, k} \neq 0$ for some $k \in\left\{1, \ldots,\left\lfloor\frac{d}{2 \ell}\right\rfloor\right\}$.

Proof. It is sufficient, by Proposition [34, to consider non-singular plane curves that is defined by equation (5). First, assume that $\beta_{2 \ell k, \ell k}=0$ for all $k=1, \ldots,\left\lfloor\frac{d}{2 \ell}\right\rfloor$, thus elements of $\widehat{M_{g}^{P l}(G)}$ have a plane model which is 
isomorphic to the form $X^{d}+Y^{d-1} Z+\alpha Y Z^{d-1}=0$. The full automorphism group in such case is well known by Proposition 15. This proves (1) and (2).

Secondly, suppose that $\beta_{2 \ell j, \ell j} \neq 0$ for some $j \in\left\{1, \ldots,\left\lfloor\frac{d}{2 \ell}\right\rfloor\right\}$. It is to be noted that the form (5) of Proposition 34 always admits a a bigger automorphism group namely, $G_{0}:=<\sigma, \tau>$ of order $2 \ell(d-2)$ where $\sigma:=$ $\left[X ; \xi_{\ell(d-2)} Y ; \xi_{\ell(d-2)}^{d-3} Z\right]$ and $\tau:=\left[X ; \mu Z ; \mu^{-1} Y\right]$ with $\mu^{d-2}=\alpha$. Consequently Aut $(C)$ is not cyclic, since $G_{0}$ does being isomorphic to $<\sigma, \tau \mid \tau^{2}=\sigma^{\ell(d-2)}=1$, and $\tau \sigma \tau=\sigma^{-(d-1)}>$. Also $C$ is not a descendant of the Klein curve $K_{d}$ because $\left|G_{0}\right| \nmid 3\left(d^{2}-3 d+3\right)$. Moreover $A u t(C)$ is not conjugate to any of the finite primitive subgroups of $P G L_{3}(K)$, since $\ell(d-2) \geq 8$ and non of these groups contains elements of order $>7$ (in fact, the Klein group $P S L(2,7)$ is the only primitive group in $P G L_{3}(K)$ with elements of order 7$)$. On the other hand, $C$ is not a descendant of the Fermat curve, since $\ell(d-2)>2 d$ for all $\ell>2$ and elements of $\operatorname{Aut}\left(F_{d}\right)$ have orders at most $2 d$ also for $\ell=2,4(d-2) \nmid 6 d^{2}$ because $d \geq 6$ and is even.

Now it follows by the above argument that $\operatorname{Aut}(C)$ should fix a line and a point off that line where the fixed point does not belong to $C$. But we have $\sigma, \tau \in A u t(C)$ therefore the line must be $X=0$ and the point is $P_{1}$. In particular, automorphisms of $C$ are of the form $\left[X ; \beta_{2}^{\prime} Y+\beta_{3}^{\prime} Z ; \gamma_{2}^{\prime} Y+\gamma_{3}^{\prime} Z\right]$ and we can think about Aut $(C)$ in a short exact sequence $1 \rightarrow N \rightarrow \operatorname{Aut}(C) \rightarrow \rho(\operatorname{Aut}(C)) \rightarrow 1$ with $N=<\operatorname{diag}\left(\xi_{d}^{r^{\prime}} ; 1 ; 1\right)>$ a cyclic group of order dividing $d, \rho(\operatorname{Aut}(C))$ is conjugate to a cyclic group $\mathbb{Z} / m \mathbb{Z}$ of order $m \leq d-1$, a Dihedral group $D_{2 m}$ where $m \mid(d-2)$ (recall that $\operatorname{diag}(-1 ; 1 ; 1) \in N)$, the alternating groups $A_{4}, A_{5}$ or the permutation group $S_{4}$ and $\rho: P B D(2,1) \hookrightarrow P G L_{2}(K)$ is the canonical map where $P B D(2,1)$ is the subgroup of $P G L_{3}(K)$ that all the entries in the third column and third row are zero except the one in the diagonal which has value 1 . It suffices to consider the case $\ell=2$, since $M_{g}^{P l}(\mathbb{Z} / \ell(d-2) \mathbb{Z}) \subseteq M_{g}^{P l}(\mathbb{Z} / 2(d-2) \mathbb{Z})$. Hence $\rho(\operatorname{Aut}(C))$ contains the element $\rho(\tau)=\left(\begin{array}{cc}0 & \mu \\ \mu^{-1} & 0\end{array}\right)$ of order 2 and the element $\rho(\sigma)=\left(\begin{array}{cc}1 & 0 \\ 0 & \xi_{2(d-2)}^{d-4}\end{array}\right)$ of order $d-2$ (only if $\left.4 \nmid d-2\right)$ and $\frac{d-2}{2}$ (otherwise) therefore $\rho(A u t(C))$ always contains a dihedral subgroup and then it is not conjugate to a cyclic group $\mathbb{Z} / m \mathbb{Z}$. Now if $4 \nmid d-2$ (resp. $4 \mid d-2$ and $d \neq 6,10)$ then $\rho(\operatorname{Aut}(C))$ has elements of order $>5$. In particular, it is not conjugate to any of the groups $A_{4}, S_{4}$ or $A_{5}$. Thus $\rho(A u t(C))$ is conjugate to $D_{2(d-2)}$ but also we have $4(d-2)|| A u t(C) \mid$ therefore 2||$N \mid$ and the case (5) is proved. It remains now to determine the full automorphism group when $d=6$ or 10 :

For $d=6$, the equation (5) in Proposition [10 become $X^{6}+Y^{5} Z+Y Z^{5}+\beta_{2,4} X^{2} Y^{2} Z^{2}=0$ with $\beta_{2,4} \neq 0$. Let $\eta \in A u t(C)$ then $\eta$ is of the form $\left[X ; \beta_{2} Y ; \gamma_{3} Z\right]$ or $\left[X ; \beta_{3} Z ; \gamma_{2} Y\right]$, since the monomials $X^{2} Y^{4}$ and $X^{2} Z^{4}$ are not in the defining equation of $C$. Hence we must have $\beta_{2}^{5} \gamma_{3}=\beta_{2} \gamma_{3}^{5}=\beta_{2}^{2} \gamma_{3}^{2}=1$, which in turns implies that $|\operatorname{Aut}(C)|=16$. Therefore Aut $(C)$ is conjugate to $<\sigma, \tau \mid \tau^{2}=\sigma^{8}=1$ and $\tau \sigma \tau=\sigma^{3}>$ with $\sigma:=\left[X ; \xi_{8} Y ; \xi_{8}^{3} Z\right]$ and $\tau:=[X ; Z ; Y]$ which is $\operatorname{SmallGroup}(16,8)$ in Gap list. By a quite similar argument, one conclude that when $d=10$, the plane non-singular model is reduced to $X^{10}+Y^{9} Z+Y Z^{9}+\beta_{6,4} X^{6} Y^{2} Z^{2}+\beta_{2,8} X^{2} Y^{4} Z^{4}$ with $\left(\beta_{6,4}, \beta_{2,8}\right) \neq(0,0)$. Also $|A u t(C)|=32$ where $A u t(C)=<\sigma, \tau>$ with $\tau:=[X ; Z ; Y]$ and $\sigma:=\left[X ; \xi_{16} Y ; \xi_{16}^{-9} Z\right]$ and hence $\operatorname{Aut}(C)$ is isomorphic to $\operatorname{SmallGroup}(32,19)$.

This completes the proof.

Corollary 36. The locus $\left.M_{g}^{P l}(\widetilde{\mathbb{Z} / \ell(d}-2) \mathbb{Z}\right)$ is always empty for any even integer $\ell \geq 2$.

Now we treat the situation for which $\ell$ is odd:

Proposition 37. Suppose that $\ell \geq 2$ is an odd integer such that $\ell \mid d$ with $d \geq 6$. Any non-singular plane model of $\delta \in M_{g}^{P l}(\mathbb{Z} / \ell(d-2) \mathbb{Z})$ is $K$-isomorphic to the form

$$
X^{d}+Y^{d-1} Z+\alpha Y Z^{d-1}+\sum_{k=1}^{n} \beta_{2 \ell k, \ell k} X^{d-2 \ell k} Y^{\ell k} Z^{\ell k}=0,
$$

where $n=\frac{d}{2 \ell}$ if $d$ is even and $\left\lfloor\frac{d-1}{2 \ell}\right\rfloor$ otherwise. In particular, the loci $M_{g}^{P l}(\mathbb{Z} / \ell(d-2) \mathbb{Z})$ are ES-irreducible.

Proof. Again, by Lemma [33, any plane non-singular model of $\delta$ is $K$-isomorphic to type $\ell(d-2),(a, b)$ of the form (4.1) of Theorem 6 for some $(a, b) \in \Gamma_{\ell(d-2)}$ and $\ell(d-1) \mid(d-1) a+b, a+(d-1) b$. In particular, $2 a=(d-2) k_{0}^{\prime}+\ell k_{0}$ and $2 b=(d-2) k_{0}^{\prime}-\ell k_{0}$ for some integers $k_{0}$ and $k_{0}^{\prime}$ and we distinguish between whether 
$d$ is even or odd as follows: If $d$ is even then so is $k_{0}$ and $a=\ell k+\left(\frac{d-2}{2}\right) k^{\prime}, b=-\ell k+\left(\frac{d-2}{2}\right) k^{\prime}$ for some integers $k$ and $k^{\prime}$. Moreover $\ell \mid \frac{d}{2} k^{\prime}$, since $\ell(d-2) \mid(d-1) a+b$ and consequently $\left[X ; \xi_{\ell(d-2)} Y ; \xi_{\ell(d-2)}^{(\ell-1)(d-2)-1} Z\right]^{\ell k+\left(\frac{d-2}{2}\right) k^{\prime}}=$ $\left[X ; \xi_{\ell(d-2)}^{a} Y ; \xi_{\ell(d-2)}^{b} Z\right]$. Therefore $a=1$ and $b=(\ell-1)(d-2)-1$ is a generator of the set of solutions of the system. As usual, it remains to determine the sets $S_{2}^{d, X}$ and $S(2)^{j, X}$ for $j=2, \ldots, d-1$ with $m=\ell(d-2), a=1$ and $b=(\ell-1)(d-2)-1$. In fact these sets are the same as seen in the proof of Proposition 34 and the rest will be typical except possibly we use the automorphism $\left[X ; \xi_{\ell(d-2)} Y ; \xi_{\ell(d-2)}^{-(d-1)} Z\right]$ instead of $\left[X ; \xi_{\ell(d-2)} Y ; \xi_{\ell(d-2)}^{d-3} Z\right]$ to obtain the required equation in this case. If $d$ is odd then $k_{0}$ and $k_{0}^{\prime}$ have the same parity and $a=$ $\frac{1}{2}\left(\ell k_{0}+k_{0}^{\prime}(d-2)\right), b=\frac{1}{2}\left(-\ell k_{0}+k_{0}^{\prime}(d-2)\right)$. Also $2 \mid \pm k_{0}+\left(\frac{d}{\ell}\right) k_{0}^{\prime}$, since $\ell(d-2) \mid(d-1) a+b, a+(d-1) b$ and in particular, we can replace $k_{0}$ by $2 k-\left(\frac{d}{\ell}\right) k_{0}^{\prime}$ for some integer $k$. Consequently $\xi_{\ell(d-2)}^{b}=\xi_{\ell(d-2)}^{-(d-1) a}$ and $\left[X ; \xi_{\ell(d-2)} Y ; \xi_{\ell(d-2)}^{-(d-1)} Z\right]^{a}=\left[X ; \xi_{\ell(d-2)}^{a} Y ; \xi_{\ell(d-2)}^{b} Z\right]$. Hence $a=1$ and $b=(\ell-1)(d-2)-1$ is again a generator of the set of solutions. Finally, the sets $S_{2}^{d, X}$ and $S(2)^{j, X}$ for $j=2, \ldots, d-1$ with $m=\ell(d-2), a=1$ and $b=(\ell-1)(d-2)-1$ are given below:

$$
\begin{aligned}
S_{2}^{d, X} & :=\{i: 2 \leq i \leq d-2 \text { and } \ell(d-2) \mid i+(d-i)((\ell-1)(d-2)-1)\} \\
& =\left\{i: 2 \leq i \leq d-2 \text { and } d-2 \mid \frac{d}{\ell}(i-1)\right\} \\
& =\emptyset
\end{aligned}
$$

The last inclusion can be easily deduced because $0<\frac{d}{\ell}(i-1)<\frac{d}{\ell}(d-2)$. Therefore $\frac{d}{\ell}(i-1)=\mu(d-2)$ for some $1 \leq \mu \leq \frac{d}{\ell}-1$. This in turns gives $\frac{d}{\ell} \mid \mu$ (since $\frac{d}{\ell}$ is odd) which is not possible. Also, we have

$$
\begin{aligned}
S(2)^{j, X} & :=\{i: 0 \leq i \leq j \text { and } \ell(d-2) \mid i+(j-i)((\ell-1)(d-2)-1)\} \\
& =\{i: 0 \leq i \leq j \text { and } \ell(d-2) \mid(d-1) j-d i\} \\
& \subseteq\{i: 0 \leq i \leq j \text { and }(d-2) \mid j-2 i\}
\end{aligned}
$$

Because $|j-2 i| \leq d-1$ therefore $j-2 i=0, \pm(d-2)$ and $S(2)_{m,(a, b)}^{j, X}= \begin{cases}\emptyset, & \text { if } j \in\{1,3, \ldots, d-4\} \\ \{0, d-2\}, & \text { if } j=d-2 \\ \left\{\frac{j}{2}\right\} & \text { otherwise }\end{cases}$

Moreover we obtain the form

$$
X^{d}+Y^{d-1} Z+\alpha Y Z^{d-1}+X^{2}\left(\beta_{d-2,0} Z^{d-2}+\beta_{d-2, d-2} Y^{d-2}\right)+\sum_{j=2,4, \ldots, d-1} \beta_{j, \frac{j}{2}} X^{d-j} Y^{\frac{j}{2}} Z^{\frac{j}{2}}=0
$$

But $\left[X ; \xi_{\ell(d-2)} Y ; \xi_{\ell(d-2)}^{-(d-1)} Z\right] \in A u t(C)$ then $\beta_{d-2,0}=\beta_{d-2, d-2}=0$ moreover $\beta_{j, \frac{j}{2}}=0$ if $\ell \nmid \frac{j}{2}$.

This completes the proof.

The full automorphism group of the elements of the locus $M_{g}^{P l}(\mathbb{Z} / \ell(d-2) \mathbb{Z})$ with $\ell \geq 3$ odd is determined by the result:

Proposition 38. Let $\ell \geq 3$ be an odd integer such that $\ell \mid d$ with $d \geq 6$ and let $G$ be a finite group inside $P G L_{3}(K)$. Then $\delta \in M_{g}^{P l}(\mathbb{Z} / \ell(d-2) \mathbb{Z}) \cap \widehat{M_{g}^{P l}(G)}$ only if one of the following situations occurs:

(1) $d=6$ and $G$ is conjugate to a central extension of $S_{4}$ by $\mathbb{Z} / 6 \mathbb{Z}$. In this case, $G$ is of order 144 and $\widehat{M_{g}^{P l}(G)}$ is an irreducible set that is given by the single element $X^{6}+Y^{5} Z+Y Z^{5}=0$.

(2) $d>6$ and $G$ is conjugate to $\langle\sigma, \tau| \tau^{2}=\sigma^{d(d-2)}=1, \tau \sigma \tau=\sigma^{-(d-1)}>$, a central extension of order $2 d(d-2)$ of $D_{2(d-2)}$ by $\mathbb{Z} / d \mathbb{Z}$. Also $\widehat{M_{g}^{P l}(G)}$ is an irreducible set and is given by one element with a non-singular plane model isomorphic to $X^{d}+Y^{d-1} Z+Y Z^{d-1}=0$.

(3) $\ell=5, d=10$ and $G$ is conjugate to $\operatorname{SmallGroup}(80,25)$. In this case every $\delta \in M_{36}^{P l}(\operatorname{SmallGroup}(80,25))$ has a non-singular plane model which is $K$-equivalent to $X^{10}+Y^{9} Z+Y Z^{9}+\beta_{10,5} Y^{5} Z^{5}=0$ with $\beta_{10,5} \neq 0$.

(4) $\ell>3, d \neq 10$ and $G$ is an element of $\operatorname{Ext}^{1}\left(N, D_{2 m}\right)$ where $N$ is a cyclic group order dividing $d$ and $m=d-2$ with $2 \nmid d$ and $\ell|| N \mid$ or $m=\frac{d-2}{2}$ with $2 \mid d$ and $2 \ell|| N \mid$. Moreover $G$ contains a subgroup which is isomorphic to $\left\langle\sigma, \tau: \tau^{2}=\sigma^{\ell(d-2)}=1\right.$ and $\tau \sigma \tau=\sigma^{-(d-1)}>$ as a subgroup. Also, every 
element $\delta \in \widehat{M_{g}^{P l}(G)}$ has a plane model that is $K$ - equivalent to (6) such that $\beta_{2 \ell j, \ell j} \neq 0$ for some $j \in\{1,2, \ldots, n\}$.

Proof. We could apply the same argument of Proposition 35 to conclude the following:

- Case (1) or (2) occurs if and only if $\beta_{2 \ell k, \ell k}=0$ for all $k \in\{1,2, \ldots, n\}$.

- Every plane non-singular model $C$ of $\delta \in M_{g}^{P l}(\mathbb{Z} / \ell(d-2) \mathbb{Z})$ (which is isomorphic to equation (6) $)$ admits always $G_{0}$ as a subgroup of order $2 \ell(d-2)$ with $\sigma:=\left[X ; \xi_{\ell(d-2)} Y ; \xi_{\ell(d-2)}^{-(d-1)} Z\right]$ and $\tau:=\left[X ; \mu Z ; \mu^{-1} Y\right]$ where $\mu^{d-2}=\alpha$. In particular, $\operatorname{Aut}(\delta)$ is not cyclic

- $\delta$ is not a descendant of the the Klein curve and also $A u t(\delta)$ is not conjugate to any of the finite primitive groups inside $P G L_{3}(K)$.

- If $\ell \neq 3$ or $d \neq 6, \delta$ is not a descendant of the Fermat curve.

Assuming that $\ell \neq 3$ or $d \neq 6$ and following the same ideas, we can think about $A u t(C)$ in a short exact sequence $1 \rightarrow N \rightarrow$ Aut $(C) \rightarrow \rho(\operatorname{Aut}(C)) \rightarrow 1$ where $\rho(\operatorname{Aut}(C))$ contains the element $\rho(\tau)=\left(\begin{array}{cc}0 & \mu \\ \mu^{-1} & 0\end{array}\right)$ of order 2 and the element $\rho(\sigma)=\left(\begin{array}{cc}1 & 0 \\ 0 & \xi_{d-2}^{\frac{d}{\ell}}\end{array}\right)$ of order $d-2$ (only if $2 \nmid d$ ) and $\frac{d-2}{2}$ (otherwise). In particular, $\rho(A u t(C))$ is not cyclic. Moreover, if $2 \nmid d$ (resp. $2 \mid d$ and $d \neq 10)$ then $\rho(A u t(C))$ is not conjugate to $A_{4}, S_{4}$ or $A_{5}$, since it has an element of order $>5$. Consequently $\rho(A u t(C))$ is conjugate to $D_{2(d-2)}$ or $D_{2\left(\frac{d-2}{2}\right)}($ only if $2 \mid d)$ and $|A u t(C)|=2(d-2)|| N \mid$ or $(d-2)|N|$. Therefore $|N|$ should be divisible by $\ell$ or $2 \ell$, since $2 \ell(d-2)|| A u t(C) \mid$.

If $d=10$ then $\ell=5$ and the equation (6) in Proposition 37 is reduced to $X^{10}+Y^{9} Z+Y Z^{9}+\beta_{1} Y^{5} Z^{5}=0$. Also $N=<\operatorname{diag}\left(\xi_{10} ; 1 ; 1\right)>$ and $\rho(A u t(C))$ is not conjugate to $A_{4}$ or $A_{5}$, since $D_{8} \preceq \rho(A u t(C))$. Therefore $\rho(A u t(C))$ is conjugate to $S_{4}, D_{16}$ or $D_{8}$. If $\rho(A u t(C)) \equiv S_{4}$ then there exists an element $\tau^{\prime} \in P G L_{2}(K)$ such that $\rho(\sigma)^{2} \tau^{\prime}$ and $\tau^{\prime-1} \rho(\tau) \rho(\sigma)^{2}$ are of order 2 and moreover $\rho(\sigma)^{2}\left(\rho(\tau) \tau^{\prime} \rho(\tau)\right)=\rho(\tau) \rho(\sigma)^{2} \tau^{\prime}$. The first relation gives $\tau^{\prime}=\left(\begin{array}{cc}\mu_{1} & \xi_{4}^{a} \\ 1 & \mu_{1}\end{array}\right)$, and then imposing the second condition to get $\exists \lambda \in K^{*}$ such that $\lambda \mu=-\mu_{1}, \lambda \mu_{1}=$ $-\mu, \lambda \mu_{1}=\mu^{-1} \xi_{4}^{a}$ and $\lambda \mu^{-1} \xi_{4}^{a}=\mu_{1}$ hence $-1=\lambda^{2}=1$ a contradiction. If $\rho(A u t(C)) \equiv D_{16}$ then there must be an element $\tau^{\prime} \in P G L_{2}(K)$ of order 2 such that $\tau^{\prime} \rho(\sigma)^{2}$ has order 8 with $\rho(\tau), \rho(\sigma) \in<\tau^{\prime}, \rho(\sigma)^{2}>=D_{16}$. In particular $\left(\tau^{\prime} \rho(\sigma)^{2}\right)^{2}=\rho(\sigma)$ or $\rho(\sigma)^{-1}$ (being the only elements of order 4 inside $\left.D_{16}\right)$ hence $\tau^{\prime}=\left(\begin{array}{cc}0 & \mu_{2} \\ \mu_{3} & 0\end{array}\right)$. In this case $\tau^{\prime} \tau^{\prime} \rho(\sigma)^{2}$ is of order $2<8$ a contradiction. We then conclude that $\rho(A u t(C))$ is conjugate to $D_{8}$ and $|A u t(C)|=80$. More precisely, $\operatorname{Aut}(C)$ is generated by $\sigma:=\left[X ; \xi_{40} Y ; \xi_{40}^{-9} Z\right]$ and $\tau:=[X ; Z ; Y]$ which is isomorphic to $\left\langle\sigma, \tau: \tau^{2}=\sigma^{40}=1\right.$ and $\tau \sigma \tau=\sigma^{-9}>\cong \operatorname{SmallGroup}(80,25)$.

Finally it remains to treat the case $\ell=3$ and $d=6$ where $C: X^{6}+Y^{5} Z+Y Z^{5}+\beta_{1} Y^{3} Z^{3}=0$ is a descendant of the Fermat sextic curve through a transformation $P \in P G L_{3}(K)$. Since $C$ admits an automorphism $\sigma:=\left[X ; \xi_{12} Y ; \xi_{12}^{-5} Z\right]$ of order 12 then $\sigma^{4}=[\omega X ; Y ; Z] \in A u t(C)$ is a homology of order 3 . Also homologies of order 3 inside $A u t\left(F_{6}\right)$ are divided into $S_{1}:=\{[\omega X ; Y ; Z],[X ; \omega Y ; Z],[X ; Y ; \omega Z]\}$ and $S_{2}:=\left\{\left[\omega^{2} X ; Y ; Z\right],\left[X ; \omega^{2} Y ; Z\right],\left[X ; Y ; \omega^{2} Z\right]\right\}$ where both sets lie in different conjugacy classes in $P G L_{3}(K)$. Consequently $P^{-1} \sigma^{4} P \in S_{1}$ and because the elements of $S_{1}$ are conjugate to each others inside $A u t\left(F_{6}\right)$, we need only to consider the situation $P^{-1} \sigma^{4} P=\sigma^{4}$. Thus $P=\left[X ; \mu_{2} Y+\mu_{3} Z ; \gamma_{2} Y+\gamma_{3} Z\right]$ and $C$ is transformed to the form $\widehat{C}: X^{6}+\nu_{0} Y^{6}+\nu_{1} Z^{6}+G(Y, Z)$ where $\nu_{0}:=\gamma_{2} \mu_{2}\left(\gamma_{2}^{4}+\beta \mu_{2}^{2} \gamma_{2}^{2}+\mu_{2}^{4}\right)(=1)$ and $\nu_{1}:=\gamma_{3} \mu_{3}\left(\gamma_{3}^{4}+\beta \mu_{3}^{2} \gamma_{3}^{2}+\mu_{3}^{4}\right)(=1)$. In particular, $\left(\gamma_{2} \mu_{2}\right)\left(\gamma_{3} \mu_{3}\right) \neq 0$ and $\left[\xi_{6}^{b} Y ; \xi_{6}^{a} X ; Z\right],\left[\xi_{6}^{b} Z ; Y ; \xi_{6}^{a} X\right] \notin$ $\operatorname{Aut}(\widehat{C})$. Hence $P^{-1} \sigma P=\left[X ; Z ; \xi_{6}^{b} Y\right] \in A u t(\widehat{C})$ with $b=1$ or 5 , since elements of order 12 in $A u t\left(F_{6}\right)$ are $\left[X ; \xi_{6}^{a} Z ; \xi_{6}^{b} Y\right],\left[\xi_{6}^{b} Y ; \xi_{6}^{a} X ; Z\right]$ or $\left[\xi_{6}^{b} Z ; Y ; \xi_{6}^{a} X\right]$ such that $\operatorname{gcd}(6, a+b)=1$ and moreover any such element is conjugate inside $A u t\left(F_{6}\right)$ to $\left[X ; Z ; \xi_{6}^{b} Y\right]$ with $b=1$ or 5 . On the other hand, $P^{-1} \tau P \in A u t(\widehat{C})$ is of order 2 thus $\mu_{3}=\mu_{2}, \gamma_{3}=\gamma_{2}$ or $\mu_{3}=-\mu_{2}, \gamma_{3}=-\gamma_{2}$, which in turns reduces $\widehat{C}$ to $X^{6}+(Y \pm Z)^{6}$. This is not possible because $\left[X ; Z ; \xi_{6}^{b} Y\right]$ with $b=1$ or 5 does not retain $\widehat{C}$, therefore $C$ is not be a descendant of the Fermat curve. This completes the proof. 


\section{Appendix A. Tables of Type $m(a, b)$ For Degree $d \leq 9$}

In this appendix we introduce tables for the types of cyclic groups and the equations that are obtained as a result of $\S 2$ with respect to low degrees. In particular, we list the possible $m,(a, b)$ such that $\rho_{m, a, b}\left(M_{g}^{P l}(\mathbb{Z} / m)\right)$ may be non-trivial, and we associate a normal form $F(X ; Y ; Z)=0$ for such loci, where any element of the locus has a plane non-singular model for some specialization of the parameters. The notation of the parameters, for a fixed degree $d$, are unrelated from one type to another one: for example, we use, by an abuse of notation, $\beta_{i, j}$ as the parameter of the monomial $X^{d-j} Y^{i} Z^{j-i}$ in any normal form.

It might happen that two types $m,(a, b)$ and $m,\left(a^{\prime}, b^{\prime}\right)$ are isomorphic through a permutation of the variables or $F(X ; Y ; Z)$ decomposes into a product $X . G(X ; Y ; Z)$. The following tables are obtained by compiling the SAGE code of Theorem [6] and then removing those types which are isomorphic to a certain type or are not irreducible, see the programm in http://mat.uab.cat/ eslam/CAGPC.sagews

TABLE 1. Quartics

\begin{tabular}{|c|c|}
\hline Type: $m,(a, b)$ & $F(X ; Y ; Z)$ \\
\hline \hline $12,(3,4)$ & $X^{4}+Y^{4}+\alpha X Z^{3}$ \\
\hline $9,(1,6)$ & $X^{4}+Y^{3} Z+\alpha X Z^{3}$ \\
\hline $8,(1,5)$ & $X^{4}+Y^{3} Z+\alpha Y Z^{3}$ \\
\hline $7,(1,5)$ & $X^{3} Y+Y^{3} Z+\alpha Z^{3} X$ \\
\hline $6,(3,4)$ & $X^{4}+Y^{4}+\alpha X Z^{3}+\beta_{2,2} X^{2} Y^{2}$ \\
\hline $4,(1,2)$ & $X^{4}+Y^{4}+Z^{4}+\beta_{2,0} X^{2} Z^{2}+\beta_{3,2} X Y^{2} Z$ \\
\hline $4,(0,1)$ & $Z^{4}+L_{4, Z}$ \\
\hline $3,(1,2)$ & $X^{4}+X\left(Z^{3}+\alpha Y^{3}\right)+\beta_{2,1} X^{2} Y Z+\beta_{4,2} Y^{2} Z^{2}$ \\
\hline $3,(0,1)$ & $Z^{3} L_{1, Z}+L_{4, Z}$ \\
\hline $2,(0,1)$ & $Z^{4}+Z^{2} L_{2, Z}+L_{4, Z}$ \\
\hline
\end{tabular}

TABLE 2. Quintics

\begin{tabular}{|c|c|}
\hline Type: $m,(a, b)$ & $F(X ; Y ; Z)$ \\
\hline \hline $20,(4,5)$ & $X^{5}+Y^{5}+\alpha X Z^{4}$ \\
\hline $16,(1,12)$ & $X^{5}+Y^{4} Z+\alpha X Z^{4}$ \\
\hline $15,(1,11)$ & $X^{5}+Y^{4} Z+\alpha Y Z^{4}$ \\
\hline $13,(1,10)$ & $X^{4} Y+Y^{4} Z+\alpha Z^{4} X$ \\
\hline $10,(2,5)$ & $X^{5}+Y^{5}+\alpha X Z^{4}+\beta_{2,0} X^{3} Z^{2}$ \\
\hline $8,(1,4)$ & $X^{5}+Y^{4} Z+\alpha X Z^{4}+\beta_{2,0} X^{3} Z^{2}$ \\
\hline $5,(1,2)$ & $X^{5}+Y^{5}+Z^{5}+\beta_{3,1} X^{2} Y Z^{2}+\beta_{4,3} X Y^{3} Z$ \\
\hline $5,(0,1)$ & $Z^{5}+L_{5, Z}$ \\
\hline $4,(1,2)$ & $X^{5}+X\left(Z^{4}+\alpha Y^{4}\right)+\beta_{2,0} X^{3} Z^{2}+\beta_{3,2} X^{2} Y^{2} Z+\beta_{5,2} Y^{2} Z^{3}$ \\
\hline
\end{tabular}




\begin{tabular}{|c|c|}
\hline $4,(0,1)$ & $Z^{4} L_{1, Z}+L_{5, Z}$ \\
\hline $3,(1,2)$ & $X^{5}+Y^{4} Z+\alpha Y Z^{4}+\beta_{2,1} X^{3} Y Z+X^{2}\left(\beta_{3,0} Z^{3}+\beta_{3,3} Y^{3}\right)+\beta_{4,2} X Y^{2} Z^{2}$ \\
\hline $2,(0,1)$ & $Z^{4} L_{1, Z}+Z^{2} L_{3, Z}+L_{5, Z}$ \\
\hline
\end{tabular}

Table 3. Sextics

\begin{tabular}{|c|c|}
\hline Type: $m,(a, b)$ & $F(X ; Y ; Z)$ \\
\hline \hline $30,(5,6)$ & $X^{6}+Y^{6}+\alpha X Z^{5}$ \\
\hline $25,(1,20)$ & $X^{6}+Y^{5} Z+\alpha X Z^{5}$ \\
\hline $24,(1,19)$ & $X^{6}+Y^{5} Z+\alpha Y Z^{5}$ \\
\hline $21,(1,17)$ & $X^{5} Y+Y^{5} Z+\alpha X Z^{5}$ \\
\hline $15,(5,6)$ & $X^{6}+Y^{6}+\alpha X Z^{5}+\beta_{3,3} X^{3} Y^{3}$ \\
\hline $12,(1,7)$ & $X^{6}+Y^{5} Z+\alpha Y Z^{5}+\beta_{6,3} Y^{3} Z^{3}$ \\
\hline $10,(5,6)$ & $X^{6}+Y^{6}+\alpha X Z^{5}+\beta_{2,2} X^{4} Y^{2}+\beta_{4,4} X^{2} Y^{4}$ \\
\hline $8,(1,3)$ & $X^{6}+Y^{5} Z+\alpha Y Z^{5}+\beta_{4,2} X^{2} Y^{2} Z^{2}$ \\
\hline $6,(1,2)$ & $X^{6}+Y^{6}+Z^{6}+\beta_{3,0} X^{3} Z^{3}+\beta_{4,2} X^{2} Y^{2} Z^{2}+\beta_{5,4} X Y^{4} Z$ \\
\hline $6,(1,3)$ & $X^{6}+Y^{6}+Z^{6}+\beta_{2,0} X^{4} Z^{2}+\beta_{6,3} Y^{3} Z^{3}+X^{2}\left(\beta_{4,0} Z^{4}+\beta_{4,3} Y^{3} Z\right)$ \\
\hline $6,(0,1)$ & $X^{6}+X Z^{5}+\alpha X Y^{5}+\beta_{3,1} X^{3} Y Z^{2}+\beta_{4,3} X^{2} Y^{3} Z+\beta_{6,2} Y^{2} Z^{4}$ \\
\hline $5,(1,2)$ & $X^{6}+X Z^{5}+\alpha X Y^{5}+\beta_{2,1} X^{4} Y Z+\beta_{4,2} X^{2} Y^{2} Z^{2}+\beta_{6,3} Y^{3} Z^{3}$ \\
\hline $5,(1,4)$ & $Z^{5} L_{1, Z}+L_{6, Z}$ \\
\hline $5,(0,1)$ & $Z^{6}+Z^{3} L_{3, Z}+L_{6, Z}$ \\
\hline $4,(1,3)$ & $X^{6}+Y^{5} Z+\alpha Y Z^{5}+\beta_{6,3} Y^{3} Z^{3}+\beta_{2,1} X^{4} Y Z+X^{2}\left(\beta_{4,0} Z^{4}+\beta_{4,2} Y^{2} Z^{2}+\beta_{4,4} Y^{4}\right)$ \\
\hline $3,(0,1)$ & \\
\hline $2,(0,1)$ & \\
\hline
\end{tabular}

TABLe 4 . degree 7

\begin{tabular}{|c|c|}
\hline Type: $m,(a, b)$ & $F(X ; Y ; Z)$ \\
\hline \hline $42,(6,7)$ & $X^{7}+Y^{7}+\alpha X Z^{6}$ \\
\hline $36,(1,30)$ & $X^{7}+Y^{6} Z+\alpha X Z^{6}$ \\
\hline $35,(1,29)$ & $X^{7}+Y^{6} Z+\alpha Y Z^{6}$ \\
\hline $31,(1,26)$ & $X^{6} Y+Y^{6} Z+\alpha X Z^{6}$ \\
\hline $21,(3,7)$ & $X^{7}+Y^{7}+\alpha X Z^{6}+\beta_{3,0} X^{4} Z^{3}$ \\
\hline $18,(1,12)$ & $X^{7}+Y^{6} Z+\alpha X Z^{6}+\beta_{3,0} X^{4} Z^{3}$ \\
\hline $14,(2,7)$ & $X^{7}+Y^{7}+\alpha X Z^{6}+\beta_{2,0} X^{5} Z^{2}+\beta_{4,0} X^{3} Z^{4}$ \\
\hline $12,(1,6)$ & $X^{7}+Y^{6} Z+\alpha X Z^{6}+\beta_{2,0} X^{5} Z^{2}+\beta_{4,0} X^{3} Z^{4}$ \\
\hline $9,(1,3)$ & $X^{7}+Y^{6} Z+\alpha X Z^{6}+\beta_{3,0} X^{4} Z^{3}+\beta_{5,3} X^{2} Y^{3} Z^{2}$ \\
\hline $7,(1,2)$ & $X^{7}+Y^{7}+Z^{7}+\beta_{4,1} X^{3} Y Z^{3}+\beta_{5,3} X^{2} Y^{3} Z^{2}+\beta_{6,5} X Y^{5} Z$ \\
\hline $7,(1,3)$ & $X^{7}+Y^{7}+Z^{7}+\beta_{3,1} X^{4} Y Z^{2}+\beta_{5,4} X^{2} Y^{4} Z+\beta_{6,2} X Y^{2} Z^{4}$ \\
\hline $7,(0,1)$ & $Z^{7}+L_{7, Z}$ \\
\hline
\end{tabular}




\begin{tabular}{|c|c|}
\hline $6,(1,2)$ & $X^{7}+X Z^{6}+\alpha X Y^{6}+\beta_{3,0} X^{4} Z^{3}+\beta_{4,2} X^{3} Y^{2} Z^{2}+\beta_{5,4} X^{2} Y^{4} Z+\beta_{7,2} Y^{2} Z^{5}$ \\
\hline $6,(2,3)$ & $X^{7}+X Z^{6}+\alpha X Y^{6}+\beta_{2,0} X^{5} Z^{2}+\beta_{3,3} X^{4} Y^{3}+\beta_{4,0} X^{3} Z^{4}+\beta_{5,3} X^{2} Y^{3} Z^{2}+\beta_{7,3} Y^{3} Z^{4}$ \\
\hline $6,(0,1)$ & $Z^{6} L_{1, Z}+L_{7, Z}$ \\
\hline $5,(1,4)$ & $X^{7}+Y^{6} Z+\alpha Y Z^{6}+\beta_{2,1} X^{5} Y Z+\beta_{4,2} X^{3} Y^{2} Z^{2}+\beta_{6,3} X Y^{3} Z^{3}+X^{2}\left(\beta_{5,0} Z^{5}+\beta_{5,5} Y^{5}\right)$ \\
\hline $4,(1,2)$ & $X^{7}+Y^{6} Z+\alpha X Z^{6}+\beta_{2,0} X^{5} Z^{2}+\beta_{3,2} X^{4} Y^{2} Z+\beta_{5,2} X^{2} Y^{2} Z^{3}+\beta_{6,4} X Y^{4} Z^{2}+\beta_{7,2} Y^{2} Z^{5}+$ \\
& $+X^{3}\left(\beta_{4,0} Z^{4}+\beta_{4,4} Y^{4}\right)$ \\
\hline $3,(1,2)$ & $X^{7}+X Z^{6}+\alpha X Y^{6}+\beta_{2,1} X^{5} Y Z+\beta_{4,2} X^{3} Y^{2} Z^{2}+\beta_{6,3} X Y^{3} Z^{3}+\beta_{7,2} Y^{2} Z^{5}+\beta_{7,5} Y^{5} Z^{2}+$ \\
& $X^{4}\left(\beta_{3,0} Z^{3}+\beta_{3,3} Y^{3}\right)+X^{2}\left(\beta_{5,1} Y Z^{4}+\beta_{5,4} Y^{4} Z\right)$ \\
\hline $3,(0,1)$ & $Z^{6} L_{1, Z}+Z^{3} L_{4, Z}+L_{7, Z}$ \\
\hline $2,(0,1)$ & $Z^{6} L_{1, Z}+Z^{4} L_{3, Z}+Z^{2} L_{5, Z}+L_{7, Z}$ \\
\hline
\end{tabular}

TABle 5. degree 8

\begin{tabular}{|c|c|}
\hline Type: $m,(a, b)$ & $F(X ; Y ; Z)$ \\
\hline $56,(7,8)$ & $X^{8}+Y^{8}+\alpha X Z^{7}$ \\
\hline $49,(1,42)$ & $X^{8}+Y^{7} Z+\alpha X Z^{7}$ \\
\hline $48,(1,41)$ & $X^{8}+Y^{7} Z+\alpha Y Z^{7}$ \\
\hline $43,(1,37)$ & $X^{7} Y+Y^{7} Z+\alpha X Z^{7}$ \\
\hline $28,(7,8)$ & $X^{8}+Y^{8}+\alpha X Z^{7}+\beta_{4,4} X^{4} Y^{4}$ \\
\hline $24,(1,17)$ & $X^{8}+Y^{7} Z+\alpha Y Z^{7}+\beta_{8,4} Y^{4} Z^{4}$ \\
\hline $16,(1,9)$ & $X^{8}+Y^{7} Z+\alpha Y Z^{7}+\beta_{8,5} Y^{5} Z^{3}+\beta_{8,3} Y^{3} Z^{5}$ \\
\hline $14,(7,8)$ & $X^{8}+Y^{8}+\alpha X Z^{7}+\beta_{2,2} X^{6} Y^{2}+\beta_{4,4} X^{4} Y^{4}+\beta_{6,6} X^{2} Y^{6}$ \\
\hline $12,(1,5)$ & $X^{8}+Y^{7} Z+\alpha Y Z^{7}+\beta_{8,4} Y^{4} Z^{4}+\beta_{4,2} X^{4} Y^{2} Z^{2}$ \\
\hline $8,(1,2)$ & $X^{8}+Y^{8}+Z^{8}+\beta_{4,0} X^{4} Z^{4}+\beta_{5,2} X^{3} Y^{2} Z^{3}+\beta_{6,4} X^{2} Y^{4} Z^{2}+\beta_{7,6} X Y^{6} Z$ \\
\hline $8,(1,3)$ & $X^{8}+Y^{8}+Z^{8}+\beta_{4,2} X^{4} Y^{2} Z^{2}+\beta_{8,4} Y^{4} Z^{4}+X^{2}\left(\beta_{6,1} Y Z^{5}+\beta_{6,5} Y^{5} Z\right)$ \\
\hline $8,(1,4)$ & $X^{8}+Y^{8}+Z^{8}+\beta_{2,0} X^{6} Z^{2}+\beta_{4,0} X^{4} Z^{4}+\beta_{5,4} X^{3} Y^{4} Z+\beta_{6,0} X^{2} Z^{6}+\beta_{7,4} X Y^{4} Z^{3}$ \\
\hline $8,(0,1)$ & $Z^{8}+L_{8, Z}$ \\
\hline $7,(1,2)$ & $X^{8}+X Z^{7}+\alpha X Y^{7}+\beta_{4,1} X^{4} Y Z^{3}+\beta_{5,3} X^{3} Y^{3} Z^{2}+\beta_{6,5} X^{2} Y^{5} Z+\beta_{8,2} Y^{2} Z^{6}$ \\
\hline $7,(1,3)$ & $X^{8}+X Z^{7}+\alpha X Y^{7}+\beta_{3,1} X^{5} Y Z^{2}+\beta_{5,4} X^{3} Y^{4} Z+\beta_{6,2} X^{2} Y^{2} Z^{4}+\beta_{8,5} Y^{5} Z^{3}$ \\
\hline $7,(1,6)$ & $X^{8}+X Z^{7}+\alpha X Y^{7}+\beta_{2,1} X^{6} Y Z+\beta_{4,2} X^{4} Y^{2} Z^{2}+\beta_{6,3} X^{2} Y^{3} Z^{3}+\beta_{8,4} Y^{4} Z^{4}$ \\
\hline $7,(0,1)$ & $Z^{7} L_{1, Z}+L_{8, Z}$ \\
\hline $6,(1,5)$ & $\begin{aligned} X^{8}+Y^{7} Z+ & \alpha Y Z^{7}+\beta_{2,1} X^{6} Y Z+\beta_{4,2} X^{4} Y^{2} Z^{2}+\beta_{8,4} Y^{4} Z^{4} \\
& +X^{2}\left(\beta_{6,0} Z^{6}+\beta_{6,3} Y^{3} Z^{3}+\beta_{6,6} Y^{6}\right)\end{aligned}$ \\
\hline $4,(0,1)$ & $Z^{8}+Z^{4} L_{4, Z}+L_{8, Z}$ \\
\hline $3,(1,2)$ & $\begin{array}{c}X^{8}+Y^{7} Z+\alpha Y Z^{7}+\beta_{8,4} Y^{4} Z^{4}+\beta_{2,1} X^{6} Y Z+\beta_{4,2} X^{4} Y^{2} Z^{2}+X^{5}\left(\beta_{3,0} Z^{3}+\beta_{3,3} Y^{3}\right)+ \\
+X^{3}\left(\beta_{5,1} Y Z^{4}+\beta_{5,4} Y^{4} Z\right)+X^{2}\left(\beta_{6,0} Z^{6}+\beta_{6,3} Y^{3} Z^{3}+\beta_{6,6} Y^{6}\right)+X\left(\beta_{7,2} Y^{2} Z^{5}+\beta_{7,5} Y^{5} Z^{2}\right) \\
\end{array}$ \\
\hline $2,(0,1)$ & $Z^{8}+Z^{6} L_{2, Z}+Z^{4} L_{4, Z}+Z^{2} L_{6, Z}+L_{8, Z}$ \\
\hline
\end{tabular}


TABLE 6. degree 9

\begin{tabular}{|c|c|}
\hline Type: $m,(a, b)$ & $F(X ; Y ; Z)$ \\
\hline $72,(8,9)$ & $X^{9}+Y^{9}+\alpha X Z^{8}$ \\
\hline $64,(1,56)$ & $X^{9}+Y^{8} Z+\alpha X Z^{8}$ \\
\hline $63,(1,55)$ & $X^{9}+Y^{8} Z+\alpha Y Z^{8}$ \\
\hline $57,(1,50)$ & $X^{8} Y+Y^{8} Z+\alpha X Z^{8}$ \\
\hline $36,(4,9)$ & $X^{9}+Y^{9}+\alpha X Z^{8}+\beta_{4,0} X^{5} Z^{4}$ \\
\hline $32,(1,24)$ & $X^{9}+Y^{8} Z+\alpha X Z^{8}+\beta_{4,0} X^{5} Z^{4}$ \\
\hline $24,(8,9)$ & $X^{9}+Y^{9}+\alpha X Z^{8}+\beta_{3,3} X^{6} Y^{3}+\beta_{6,6} X^{3} Y^{6}$ \\
\hline $21,(1,13)$ & $X^{9}+Y^{8} Z+\alpha Y Z^{8}+\beta_{6,3} X^{3} Y^{3} Z^{3}$ \\
\hline $18,(2,9)$ & $X^{9}+Y^{9}+\alpha X Z^{8}+\beta_{2,0} X^{7} Z^{2}+\beta_{4,0} X^{5} Z^{4}+\beta_{6,0} X^{3} Z^{6}$ \\
\hline $16,(1,8)$ & $X^{9}+Y^{8} Z+\alpha X Z^{8}+\beta_{2,0} X^{7} Z^{2}+\beta_{4,0} X^{5} Z^{4}+\beta_{6,0} X^{3} Z^{6}$ \\
\hline $12,(4,9)$ & $X^{9}+Y^{9}+\alpha X Z^{8}+\beta_{3,3} X^{6} Y^{3}+\beta_{4,0} X^{5} Z^{4}+\beta_{6,6} X^{3} Y^{6}+\beta_{7,3} X^{2} Y^{3} Z^{4}$ \\
\hline $9,(1,2)$ & $X^{9}+Y^{9}+Z^{9}+\beta_{5,1} X^{4} Y Z^{4}+\beta_{6,3} X^{3} Y^{3} Z^{3}+\beta_{7,5} X^{2} Y^{5} Z^{2}+\beta_{8,7} X Y^{7} Z$ \\
\hline $9,(1,3)$ & $X^{9}+Y^{9}+Z^{9}+\beta_{3,0} X^{6} Z^{3}+\beta_{5,3} X^{4} Y^{3} Z^{2}+\beta_{6,0} X^{3} Z^{6}+\beta_{7,6} X^{2} Y^{6} Z+\beta_{8,3} X Y^{3} Z^{5}$ \\
\hline $9,(0,1)$ & $Z^{9}+L_{9, Z}$ \\
\hline $8,(1,2)$ & $X^{9}+X Z^{8}+\alpha X Y^{8}+\beta_{4,0} X^{5} Z^{4}+\beta_{5,2} X^{4} Y^{2} Z^{3}+\beta_{6,4} X^{3} Y^{4} Z^{2}+\beta_{7,6} X^{2} Y^{6} Z+\beta_{9,2} Y^{2} Z^{7}$ \\
\hline $8,(1,4)$ & $X^{9}+X Z^{8}+\alpha X Y^{8}+\beta_{2,0} X^{7} Z^{2}+\beta_{4,0} X^{5} Z^{4}+\beta_{5,4} X^{4} Y^{4} Z+\beta_{6,0} X^{3} Z^{6}+\beta_{7,4} X^{2} Y^{4} Z^{3}+\beta_{9,4} Y^{4} Z^{5}$ \\
\hline $8,(1,6)$ & $X^{9}+X Z^{8}+\alpha X Y^{8}+\beta_{3,2} X^{6} Y^{2} Z+\beta_{4,0} X^{5} Z^{4}+\beta_{6,4} X^{3} Y^{4} Z^{2}+\beta_{7,2} X^{2} Y^{2} Z^{5}+\beta_{9,6} Y^{6} Z^{3}$ \\
\hline $8,(0,1)$ & $Z^{8} L_{1, Z}+L_{9, Z}$ \\
\hline $7,(1,6)$ & $\begin{array}{c}X^{9}+Y^{8} Z+\alpha Y Z^{8}+\beta_{2,1} X^{7} Y Z+\beta_{4,2} X^{5} Y^{2} Z^{2}+\beta_{6,3} X^{3} Y^{3} Z^{3}+ \\
+\beta_{8,4} X Y^{4} Z^{4}+X^{2}\left(\beta_{7,0} Z^{7}+\beta_{7,7} Y^{7}\right)\end{array}$ \\
\hline $6,(2,3)$ & $\begin{array}{c}X^{9}+Y^{9}+\alpha X Z^{8}+\beta_{2,0} X^{7} Z^{2}+\beta_{3,3} X^{6} Y^{3}+\beta_{4,0} X^{5} Z^{4}+\beta_{5,3} X^{4} Y^{3} Z^{2}+ \\
+\beta_{7,3} X^{2} Y^{3} Z^{4}+\beta_{7,3} Y^{3} Z^{6}+\beta_{8,6} Y^{6} Z^{3}+X^{3}\left(\beta_{6,0} Z^{6}+\beta_{6,6} Y^{6}\right) \\
\end{array}$ \\
\hline $4,(1,2)$ & $\begin{array}{c}X^{9}+X Z^{8}+\alpha X Y^{8}+\beta_{2,0} X^{7} Z^{2}+\beta_{3,2} X^{6} Y^{2} Z+\beta_{5,2} X^{4} Y^{2} Z^{3}+\beta_{8,4} X Y^{4} Z^{4}+ \\
\beta_{9,2} Y^{2} Z^{7}+\beta_{9,6} Y^{6} Z^{3}+X^{5}\left(\beta_{4,0} Z^{4}+\beta_{4,4} Y^{4}\right)+X^{3}\left(\beta_{6,0} Z^{6}+\beta_{6,4} Y^{4} Z^{2}\right)+X^{2}\left(\beta_{7,2} Y^{2} Z^{5}+\beta_{7,6} Y^{6} Z\right)\end{array}$ \\
\hline $4,(0,1)$ & $Z^{8} L_{1, Z}+Z^{4} L_{5, Z}+L_{9, Z}$ \\
\hline $3,(0,1)$ & $Z^{9}+Z^{6} L_{3, Z}+Z^{3} L_{6, Z}+L_{9, Z}$ \\
\hline $2,(0,1)$ & $Z^{8} L_{1, Z}+Z^{6} L_{3, Z}+Z^{4} L_{5, Z}+Z^{2} L_{7, Z}+L_{9, Z}$ \\
\hline
\end{tabular}

\section{REFERENCES}

[1] E. Badr, F.Bars; On the locus of smooth plane curves with a fixed automorphism group. Preprint. See chapter 1 in "On the automorphism group of non-singular plane curves fixing the degree", arXiv:1503.01149 (2015).

[2] E. Badr, F.Bars; The automorphism group for plane non-singular curves of degree 5, See chapter 3 in "On the automorphism group of non-singular plane curves fixing the degree", arXiv:1503.01149 (2015)

[3] F. Bars; On the automorphisms groups of genus 3 curves. Surveys in Math. and Math. Sciences, 2(2)(2012), 83-124.

[4] S. Crass; Solving the sextic by iteration: A study in complex geometry and dynamics. Experimental Mathematics 8(3)(1999), 209-240.

[5] I. Dolgachev; Classical Algebraic Geometry: a modern view, Cambridge Univ. Press 2012, see also Private Lecture Notes in: http://www.math.lsa.umich.edu/ idolga/.

[6] H. M. Farkas, I. Kra; Riemann Surfaces, GTM 71, Springer Verlag, 1980. 
[7] GAP, The GAP Group: Groups, Algorithms, and Programming, a system for computational discrete algebra (2008), available at http://www.gap-system.org Version 4.4.11.

[8] T. Harui; Automorphism groups of plane curves, arXiv: 1306.5842v2[math.AG] 7 Jun 2014

[9] P. Henn; Die Automorphismengruppen dar algebraischen Functionenkorper vom Geschlecht 3, Inagural-dissertation, Heidelberg, 1976.

[10] J. W. P. Hirschfeld, G. Korchmáros, F. Torres; Algebraic Curves over Finite Fields, Princeton Series in Applied Mathematics, 2008.

[11] A. Hurwitz; Über algebraische Gebilde mit eindeutigen Transformationen in sich, Math.Ann. 41 (1893),403-442.

[12] A. Kuribayashi, K. Komiya; On Weierstrass points and automor- phisms of curves of genus three. In: Algebraic geometry (Proc. Summer Meeting, Copenhagen 1978), LNM 732, 253-299, Springer (1979).

[13] H. Mitchell; Determination of the ordinary and modular ternary linear groups, Trans. Amer. Math. Soc. 12(2) (1911), 207242.

[14] J. Wolfart; The 'obivous' part of Belyi's theorem and Riemann surfaces with many autormorphism. In: Geometric Galois Actions, 1: Around Grothendieck's Esquisse d'un Programma (ed's: P.Lochak and L.Schneps), Cambridge University Press, 1997, Lecture Notes in Math. 242.

[15] H. Yoshihara; Function field theory of plane curves by dual curves, J. Algebra 239(1)(2001), 340-355

\section{- Eslam Badr}

Departament Matemàtiques, Edif. C, Universitat Autònoma de Barcelona, 08193 Bellaterra, Catalonia

E-mail address: eslam@mat.uab.cat

Department of Mathematics, Faculty of Science, Cairo University, Giza-Egypt

E-mail address: eslam@sci.cu.edu.eg

- Francesc Bars

Departament Matemàtiques, Edif. C, Universitat Autònoma de Barcelona, 08193 Bellaterra, Catalonia E-mail address: francesc@mat.uab.cat 